\title{
Lower bounds on the blow-up rate of the axisymmetric Navier-Stokes equations II
}

\author{
Chiun-Chuan Chen*, $\quad$ Robert M. Strain ${ }^{\dagger}, \quad$ Tai-Peng Tsai ${ }^{\ddagger}, \quad$ Horng-Tzer Yau ${ }^{\S}$
}

\begin{abstract}
Consider axisymmetric strong solutions of the incompressible Navier-Stokes equations in $\mathbb{R}^{3}$ with non-trivial swirl. Let $z$ denote the axis of symmetry and $r$ measure the distance to the $z$-axis. Suppose the solution satisfies, for some $0 \leq \varepsilon \leq 1,|v(x, t)| \leq C_{*} r^{-1+\varepsilon}|t|^{-\varepsilon / 2}$ for $-T_{0} \leq t<0$ and $0<C_{*}<\infty$ allowed to be large. We prove that $v$ is regular at time zero.
\end{abstract}

\section{Introduction}

The incompressible Navier-Stokes equations in cartesian coordinates are given by

$$
\partial_{t} v+(v \cdot \nabla) v+\nabla p=\Delta v, \quad \operatorname{div} v=0 .
$$

The velocity field is $v(x, t)=\left(v_{1}, v_{2}, v_{3}\right): \mathbb{R}^{3} \times\left[-T_{0}, 0\right) \rightarrow \mathbb{R}^{3}$ and $p(x, t): \mathbb{R}^{3} \times\left[-T_{0}, 0\right) \rightarrow \mathbb{R}$ is the pressure. It is a long standing open question to determine if solutions with large smooth initial data of finite energy remain regular for all time.

In this paper we consider the special class of solutions which are axisymmetric. This means, in cylindrical coordinates $r, \theta, z$ with $\left(x_{1}, x_{2}, x_{3}\right)=(r \cos \theta, r \sin \theta, z)$, that the solution is of the form

$$
v(x, t)=v_{r}(r, z, t) e_{r}+v_{\theta}(r, z, t) e_{\theta}+v_{z}(r, z, t) e_{z} .
$$

In this coordinate system $r=\sqrt{x_{1}^{2}+x_{2}^{2}}$. The components $v_{r}, v_{\theta}, v_{z}$ do not depend upon $\theta$ and the basis vectors $e_{r}, e_{\theta}, e_{z}$ are

$$
e_{r}=\left(\frac{x_{1}}{r}, \frac{x_{2}}{r}, 0\right), \quad e_{\theta}=\left(-\frac{x_{2}}{r}, \frac{x_{1}}{r}, 0\right), \quad e_{z}=(0,0,1) .
$$

The main result of our paper shows that axisymmetric solutions must blow up faster than the scale invariant rates which appears in Theorem 1.1 below.

For $R>0$ define $B\left(x_{0}, R\right) \subset \mathbb{R}^{3}$ as the ball of radius $R$ centered at $x_{0}$. The parabolic cylinder is $Q\left(X_{0}, R\right)=B\left(x_{0}, R\right) \times\left(t_{0}-R^{2}, t_{0}\right) \subset \mathbb{R}^{3+1}$ centered at $X_{0}=\left(x_{0}, t_{0}\right)$. If the center is the origin we use the abbreviations $B_{R}=B(0, R)$ and $Q_{R}=Q(0, R)$.

*Department of Mathematics and Taida Institute for Mathematical Sciences, National Taiwan University and National Center for Theoretical Sciences, Taipei Office, email: chchchen@math.ntu.edu.tw

$\dagger$ Harvard University, email: strain@math.harvard.edu

$\ddagger$ University of British Columbia, email: ttsai@math.ubc.ca

$\S$ Harvard University, email: htyau@math.harvard.edu 
Theorem 1.1 Let $(v, p)$ be an axisymmetric strong solution of the Navier-Stokes equations (N-S) in $D=$ $\mathbb{R}^{3} \times\left(-T_{0}, 0\right)$ with initial datum $\left.v\right|_{t=-T_{0}}=v^{0} \in H^{1 / 2}$ and $r v_{\theta}^{0}(r, z) \in L^{\infty}$. Suppose the pressure satisfies $p \in L^{5 / 3}(D)$ and $v$ is pointwise bounded by one of the following inequalities:

$$
|v(x, t)| \leq C_{*}|t|^{-1 / 2}, \quad(x, t) \in D .
$$

There is an $\varepsilon \in[0,1]$ such that

$$
|v(x, t)| \leq C_{*} r^{-1+\varepsilon}|t|^{-\varepsilon / 2}, \quad(x, t) \in D .
$$

The constant $C_{*}<\infty$ is allowed to be large. Then $v \in L^{\infty}\left(B_{R} \times\left[-T_{0}, 0\right]\right)$ for any $R>0$.

We remark that the case $\varepsilon=0$ is addressed in the appendix; our proof in that specific case was obtained after a preprint of [12] had appeared. The assumption (1.2) is a special case of (1.3) with $\varepsilon=1$; it is singled out for its importance. We also remark that the exponent $5 / 3$ for the norm of $p$ can be replaced, but it is the natural exponent occurring in the existence theory for weak solutions, see e.g. [1].

Recall the natural scaling of Navier-Stokes equations: If $(v, p)$ is a solution to (N-S), then for any $\lambda>0$ the following rescaled pair is also a solution:

$$
v^{\lambda}(x, t)=\lambda v\left(\lambda x, \lambda^{2} t\right), \quad p^{\lambda}(x, t)=\lambda^{2} p\left(\lambda x, \lambda^{2} t\right) .
$$

Suppose a solution $v(x, t)$ of the Navier-Stokes equations blows up at $X_{0}=\left(x_{0}, t_{0}\right)$. Leray [8] proved that the blow up rate in time is at least

$$
\|v(\cdot, t)\|_{L_{x}^{\infty}} \geq \epsilon\left(t_{0}-t\right)^{-1 / 2}
$$

Theorem 1.1 in particular rules out singular axisymmetric solutions satisfying the similar bound with $\epsilon$ large.

The main idea of our proof is as follows. We shall first prove that either (1.2) or (1.3) with $\varepsilon>0$ implies the following estimate:

$$
|v| \leq C_{*}\left(r^{2}-t\right)^{-1 / 2+2 \varepsilon}|t|^{-\varepsilon} r^{-2 \varepsilon} .
$$

This is the content of Section 2 and 3. Note that $\varepsilon$ in (1.5) differs from that in (1.3).

If (1.5) is satisfied for $\varepsilon=0$, the regularity of $v$ was proved in [3]. In Sections 4, 5 and 6 , we extend the proof of [3] to include the case (1.5) for $\varepsilon>0$. Instead of following De Giorgi and Moser's methods [5,10] used in [3], we now use Nash's idea [6,11] to prove the Hölder regularity (Section 5). This simplifies some iteration arguments in [3], but we still use De Georgi-Moser's method in the local maximum estimate in Section 4. The estimates we obtained in Sections 4 and 5 requires assumptions weaker than (1.5). Very recently KochNadirashvili-Seregin-Sverak [12] have sent us a manuscript that they have proved results similar to Theorem 1.1 using a different approach based on Liouville theorems.

\section{The case $|v| \leq C|t|^{-1 / 2}$}

Suppose we have $|v| \leq C|t|^{-1 / 2}$. Our goal is to replace the singularity of $t$ by singularity in $r$. We will derive this estimate from the equation for the $\theta$ component of the vorticity (2.7), which involves a source term $\partial_{z} v_{\theta}^{2} / r$. Under the assumption $|v| \leq C|t|^{-1 / 2}$, we have $v_{\theta}^{2} \sim|t|^{-1}$, singular in $t$ as $t \rightarrow 0$. This $t$ singularity can be weaken to $|t|^{-\varepsilon}$ after the time integration. Since the equation is scaling invariant, this improvement in the time singularity has to be offset by the space singularity. This will be achieved in some weak form in (2.15). Finally, we can transfer estimates on the vorticity to the velocity field and we thus obtain the estimate (1.5).

Recall that we always have the bound $\left|r v_{\theta}\right| \leq C$ (see Proposition 1 in [2]). Hence for some $C_{1}>0$

$$
\left|v_{\theta}\right| \leq C_{1} \min \left(r^{-1},|t|^{-1 / 2}\right), \quad\left|v_{r}\right|+\left|v_{z}\right| \leq C_{1}|t|^{-1 / 2} .
$$


For $p, q>0$, we will be using the notation

$$
\|v\|_{L_{t, x}^{q, p}\left(Q_{R}\right)}=\|v\|_{L_{t}^{q} L_{x}^{p}\left(Q_{R}\right)}=\|v\|_{L_{t}^{q} L_{x}^{p}}=\|v\|_{L_{t, x}^{q, p}}
$$

These are the usual $L^{q, p}$ spaces integrated over space and time. The domain will be suppressed in our notation below when there is no risk of ambiguity.

We will next consider the vorticity field $\omega=\operatorname{curl} v$ :

$$
\omega(x, t)=\omega_{r} e_{r}+\omega_{\theta} e_{\theta}+\omega_{z} e_{z},
$$

where

$$
\omega_{r}=-\partial_{z} v_{\theta}, \quad \omega_{\theta}=\partial_{z} v_{r}-\partial_{r} v_{z}, \quad \omega_{z}=\left(\partial_{r}+r^{-1}\right) v_{\theta} .
$$

We can deduce the following bounds for the $\theta$ component of vorticity.

Lemma 2.1 Suppose we have the pointwise bound

$$
|v(y, s)| \leq C_{1}|s|^{-1 / 2},
$$

in $Q_{R}(x, t)$. Then for any $\delta \in(0,1)$ we can estimate $\omega$ by

$$
\left\|\omega_{\theta}\right\|_{L_{t, x}^{3,4}} \leq C R^{3 / 4}|t|^{-2 / 3}+C R^{5 / 12}|t|^{-1 / 2}, \quad\left\|\omega_{\theta}\right\|_{L_{t, x}^{6,8}} \leq C R^{3 / 8}|t|^{-5 / 6}+C R^{-7 / 24}|t|^{-1 / 2} .
$$

where the integration is over $Q_{\delta R}(x, t)$ and the constant $C$ depends on $C_{1}$ and $\delta$.

Proof. We can rescale Lemma A.2 of [3] to get, for $\alpha, q \in(1, \infty)$ and $c=c(\delta, q, \alpha)$,

$$
\|\nabla v\|_{L_{t}^{\alpha} L_{x}^{q}\left(Q_{\delta R}\right)} \leq c\|f\|_{L_{t}^{\alpha} L_{x}^{q}\left(Q_{R}\right)}+c R^{-4+3 / q}\|v\|_{L_{t}^{\alpha} L_{x}^{1}\left(Q_{R}\right)} .
$$

Using $f=v_{i} v_{j}$ and the assumption (2.4), the first integral on the right is bounded by

$$
\left\|v^{2}\right\|_{L_{t}^{\alpha} L_{x}^{q}\left(Q_{R}\right)} \leq R^{3 / q}\left(\int_{-\infty}^{t}|\tau|^{-\alpha} d \tau\right)^{1 / \alpha}=R^{3 / q}|t|^{1 / \alpha-1} .
$$

The second term $R^{-4+3 / q}\|v\|_{L_{t}^{\alpha} L_{x}^{1}\left(Q_{R}\right)}$ is bounded by

$$
R^{-1+3 / q}\left(R^{2}|t|^{-\alpha / 2}\right)^{1 / \alpha}=R^{-1+3 / q+2 / \alpha}|t|^{-1 / 2}=R^{3 / q}|t|^{1 / \alpha-1}\left(R^{-2}|t|\right)^{1 / 2-1 / \alpha} .
$$

These show (2.5).

The following is our key lemma.

Lemma 2.2 Suppose that the velocity $v$ satisfies the bound (2.1) and $\|v\|_{L_{t}^{\infty} L_{x}^{1}} \leq C_{1}$ in $Q_{1}$. There is $\delta \in(0,1)$ such that, for any small $\varepsilon>0$ there is a constant $C_{2}>0$ so that $\left(\right.$ recall $\left.r=\left(x_{1}^{2}+x_{2}^{2}\right)^{1 / 2}\right)$

$$
|v(x, t)| \leq C_{2} r^{-1+2 \varepsilon}|t|^{-\varepsilon} \quad \text { in } Q_{\delta} .
$$


Proof. Step 1. We first bound the second moment of $\omega_{\theta}$. Denote $q=\omega_{\theta}$. Its equation can be written as

$$
\left[\partial_{t}+b \cdot \nabla-\Delta-\frac{v_{r}}{r}\right] q+\partial_{z} F=-\frac{q}{r^{2}}, \quad F=\frac{-v_{\theta}^{2}}{r} .
$$

See for instance [3]. Above the vector $b$ is a part of $v$,

$$
b=v_{r} e_{r}+v_{z} e_{z}, \quad b \cdot \nabla=v_{r} \partial_{r}+v_{z} \partial_{z} .
$$

Note that

$$
\operatorname{div} b=0, \quad \operatorname{curl} b=\omega_{\theta} e_{\theta} .
$$

The first equation for $b$ is because $b=v-v_{\theta} e_{\theta}, \operatorname{div} v=0$ and $\operatorname{div}\left(v_{\theta} e_{\theta}\right)=r^{-1} \partial_{\theta} v_{\theta}=0$. The second can be read from $(2.2),(2.3)$ with $v_{\theta}$ replaced by 0 . The term $\frac{q}{r^{2}}$ in $(2.7)$ has a good sign and will drop out in our estimates below. For any $x_{0}$ fixed with $r_{0}>0$, let $\xi(x)$ be a smooth cutoff function at $x_{0}$ with radius $R=r_{0} / 10$. For any $t$, let $\chi(x, s)=\xi(x) \eta(s)$ where $\eta(t)$ is a smooth cutoff function so that $\eta(t)=1$ and $\eta\left(t_{0}\right)=0$ with $t_{0}=t-R^{2}$. Let $B$ be the characteristic function of the ball centered at $x_{0}$ with radius $R$ and $\phi(x, s)=B(x) 1\left(t_{0} \leq s \leq t\right)$.

Multiply (2.7) by $\chi^{2} q$ and integrate in $\mathbb{R}^{3} \times\left(t_{0}, t\right)$. We get

$$
\begin{aligned}
\int_{\mathbb{R}^{3}} \frac{1}{2}|\chi q|^{2}(t) & +\int_{t_{0}}^{t} \int_{\mathbb{R}^{3}}|\nabla(\chi q)|^{2} \\
& \leq \int_{t_{0}}^{t} \int_{\mathbb{R}^{3}}\left[q^{2}\left(b \chi \cdot \nabla \chi+|\nabla \chi|^{2}+\frac{\chi^{2} v_{r}}{r}-\chi \dot{\chi}\right)+\chi F \partial_{z}(\chi q)+\chi F q \partial_{z} \chi\right],
\end{aligned}
$$

where $\dot{\chi}$ is the time derivative of $\chi$. The last term is bounded by

$$
\int_{t_{0}}^{t} \int_{\mathbb{R}^{3}} \chi F q \partial_{z} \chi \leq \int_{t_{0}}^{t} \int_{\mathbb{R}^{3}} q^{2}|\nabla \chi|^{2}+\chi^{2} F^{2} .
$$

The second term on the right hand side of equation (2.10) can be bounded by

$$
\int_{t_{0}}^{t} \int_{\mathbb{R}^{3}} \chi F \partial_{z}(\chi q) \leq \int_{t_{0}}^{t} \int_{\mathbb{R}^{3}}\left[\frac{\chi^{2} F^{2}}{2}+\frac{1}{2}|\nabla(\chi q)|^{2}\right] .
$$

Notice the support of $\chi$ has a distance at least $R$ from the $z$ axis. From the assumption on $v_{\theta}$, we have for any $0 \leq \varepsilon \leq 1$

$$
|F \chi|(x, s) \leq C R^{-2+\varepsilon}|s|^{-1 / 2-\varepsilon / 2} \chi(x, s) .
$$

Thus we have the integral bound for $\varepsilon>0$

$$
\int_{t_{0}}^{t} d s \int|F \chi|^{2}(x, s) d x \leq C R^{-1+2 \varepsilon}|t|^{-\varepsilon} .
$$

Now we can derive the following bound from (2.10):

$$
\int_{\mathbb{R}^{3}}|\chi q|^{2}(t) \leq 4 \int_{t_{0}}^{t} \int_{\mathbb{R}^{3}}\left[q^{2}\left(|b \chi \cdot \nabla \chi|+|\nabla \chi|^{2}+\frac{\chi^{2}\left|v_{r}\right|}{r}+|\dot{\chi}|\right)\right]+C R^{-1+2 \varepsilon}|t|^{-\varepsilon} .
$$


From the assumption (2.1), we also have for $s<t$

$$
|b \chi \cdot \nabla \chi| \leq C \chi|s|^{-1 / 2} R^{-1}, \quad|\dot{\chi}|+|\nabla \chi|^{2} \leq C R^{-2} \phi, \quad \frac{\chi^{2}\left|v_{r}\right|}{r} \leq C \chi^{2}|s|^{-1 / 2} R^{-1} .
$$

Thus we can bound the integral on the right hand side of (2.11) to get

$$
\int_{\mathbb{R}^{3}}|\chi q|^{2}(t) \leq \int_{t_{0}}^{t} d s \int_{\mathbb{R}^{3}}\left[s^{-1 / 2} R^{-1}+R^{-2}\right] q^{2}(s) \phi+R^{-1+2 \varepsilon}|t|^{-\varepsilon} .
$$

We now assume $|t|<R^{2}$. Thus $R^{-2} \lesssim|s|^{-1 / 2} R^{-1}$ in $\operatorname{supp} \phi$ and by Lemma 2.1,

$$
\|q \phi\|_{L_{t, x}^{3,4}} \leq R^{3 / 4}|t|^{-2 / 3} \text {. }
$$

This implies that

$$
\iint_{\mathbb{R}^{3}} d s s^{-1 / 2} R^{-1} q^{2}(s) \phi \leq R^{-1}\left\|s^{-1 / 2} \phi\right\|_{L_{t, x}^{3,2}}\left\|q^{2} \phi\right\|_{L_{t, x}^{3 / 2,2}} \leq R^{2}|t|^{-3 / 2} .
$$

Therefore, from (2.12) we have

$$
\int_{\mathbb{R}^{3}}|\chi q|^{2}(t) \leq R^{2}|t|^{-3 / 2}
$$

Let $\tilde{\chi}, \tilde{B}, \tilde{\phi}$ be the functions similar to $\chi, B, \phi$ with $R$ replaced by $c R$ for some small constant $c$, say $c=1 / 100$. Clearly, all previous results, in particular (2.12), remain true if we added tildes. We also have

$$
\int_{\mathbb{R}^{3}} q^{2}(s) \tilde{B} \leq C \int_{\mathbb{R}^{3}}|\chi q|^{2}(s) .
$$

We can now use this bound in (2.12) (the tilde version) and obtain

$$
\int_{\mathbb{R}^{3}}|\chi q|^{2}(t) \leq R|t|^{-1}
$$

Notice that (2.14) is a better estimate than (2.13). We can repeat this procedure in finite steps to show that, under the assumption $|t|<R^{2}$,

$$
\int_{\mathbb{R}^{3}}|\chi q|^{2}(t) \leq R^{-1+2 \varepsilon} t^{-\varepsilon}
$$

Assume now $|t|>R^{2}$. Thus $|s|^{-1 / 2} R^{-1} \lesssim R^{-2}$ in $\operatorname{supp} \phi$ and by Lemma 2.1,

$$
\|q \phi\|_{L_{t, x}^{3,4}} \leq R^{5 / 12}|t|^{-1 / 2}
$$

We have

$$
\iint d s R^{-2} q^{2} \phi \leq R^{-2}\|\phi\|_{L_{t, x}^{3,2}}\left\|q^{2} \phi\right\|_{L_{t, x}^{3 / 2,2}} \leq R|t|^{-1} .
$$

Thus

$$
\int|\chi q|^{2}(t) \leq R t^{-1}+R^{-1+2 \varepsilon} t^{-\varepsilon} \leq R^{-1+2 \varepsilon}|t|^{-\varepsilon},
$$


which is $(2.15)$.

Step 2. We now bound the fourth moment of $q$. Similar to the derivation of (2.10), we now have

$$
\begin{aligned}
\int_{\mathbb{R}^{3}}\left|\chi q^{2}\right|^{2}(t) & +\int_{t_{0}}^{t} \int_{\mathbb{R}^{3}}\left|\nabla\left(\chi q^{2}\right)\right|^{2} \\
& \leq \int_{t_{0}}^{t} \int_{\mathbb{R}^{3}} q^{4}\left(b \chi \cdot \nabla \chi+|\nabla \chi|^{2}+|\Delta \chi|+\frac{\chi^{2} v_{r}}{r}+|\dot{\chi}|\right)+\left|\chi q F \partial_{z}\left(\chi q^{2}\right)\right|+\left|q^{3} F \chi \partial_{z} \chi\right| .
\end{aligned}
$$

From the Schwarz inequality, we have

$$
\begin{gathered}
\chi q F \partial_{z}\left(\chi q^{2}\right) \leq \frac{1}{2} \chi^{2} q^{2} F^{2}+\frac{1}{2}\left|\nabla\left(\chi q^{2}\right)\right|^{2} . \\
q^{3} F \chi \partial_{z} \chi \leq R^{-2} \chi q^{4}+\chi R^{2} F^{4} .
\end{gathered}
$$

From (2.1), we have

$$
\iint \chi R^{2} F^{4} \leq \iint \chi R^{-8+2 \varepsilon} s^{-1-\varepsilon} \leq R^{-5+2 \varepsilon}|t|^{-\varepsilon} .
$$

From the bound on $\int \chi^{2} q^{2}$ in $(2.15)$

$$
\iint \chi^{2} q^{2} F^{2} \lesssim R^{-6} \int_{t-R^{2}}^{t} R^{-1+2 \varepsilon} t^{-\varepsilon} d s \leq R^{-5+2 \varepsilon}|t|^{-\varepsilon} .
$$

Therefore, we have

$$
\int_{\mathbb{R}^{3}}\left|\chi q^{2}\right|^{2}(t) \leq \iint_{\mathbb{R}^{3}}\left[s^{-1 / 2} R^{-1}+R^{-2}\right] q^{4} \phi+R^{-5+2 \varepsilon}|t|^{-\varepsilon} .
$$

We now assume $|t|<R^{2}$. Using the bound on $\left\|\omega_{\theta}\right\|_{L_{t, x}^{6,8}} \leq C R^{3 / 8} t^{-5 / 6}$ in (2.5), we have

$$
\int_{\mathbb{R}^{3}}\left|\chi q^{2}\right|^{2}(t) \leq R^{2}|t|^{-7 / 2}+R^{-5+2 \varepsilon}|t|^{-\varepsilon} .
$$

Now plug (2.18) into (2.17), we obtain a better result. Repeat this procedure as in Step 1 until we get

$$
\int_{\mathbb{R}^{3}}\left|\chi q^{2}\right|^{2}(t) \leq R^{-5+2 \varepsilon}|t|^{-\varepsilon},
$$

under the assumption $|t|<R^{2}$. For the other case $|t|>R^{2}$, Using Hölder and the bound $\left\|\omega_{\theta}\right\|_{L_{t, x}^{6,8}} \leq$ $C R^{-7 / 24} t^{-1 / 2}$ from (2.5) to estimate (2.17), we get

$$
\int_{\mathbb{R}^{3}}\left|\chi q^{2}\right|^{2}(t) \leq R^{-1}|t|^{-2}+R^{-5+2 \varepsilon}|t|^{-\varepsilon} \leq R^{-5+2 \varepsilon}|t|^{-\varepsilon},
$$

in one step.

Step 3. We now prove the pointwise bound (2.6) for $v$. Since we have already good estimates for $v_{\theta}$, it suffices to estimate $b$, which satisfies $(2.8),(2.9)$ with $\omega_{\theta}=q$. Let $\delta>0$ be a small number so that (2.15) 
and (2.19) are valid for $\left(x_{0}, t\right) \in Q_{8 \delta}$. Let $J(x)$ be a smooth cut-off function for the ball of radius $4 \delta$, with $J(x)=1$ for $|x| \leq 2 \delta$. Define

$$
\alpha(x)=\int \frac{1}{4 \pi|x-y|} \operatorname{curl}\left(J q e_{\theta}\right)(y) d y=\int\left(\nabla_{y} \frac{1}{4 \pi|x-y|}\right) \times\left(J q e_{\theta}\right)(y) d y .
$$

By the vector identity

$$
-\Delta b=\operatorname{curl} \operatorname{curl} b-\nabla \operatorname{div} b,
$$

the difference $b-\alpha$ is harmonic in the ball of radius $2 \delta$ and hence

$$
\|b-\alpha\|_{L^{\infty}\left(B_{\delta}\right)} \leq\|b-\alpha\|_{L^{1}\left(B_{2 \delta}\right)} \leq\|\alpha\|_{L^{1}\left(B_{2 \delta}\right)}+\|b\|_{L^{1}\left(B_{2 \delta}\right)} .
$$

The last term is bounded by order one since $v$ is in $L_{t}^{\infty} L_{x}^{1}$. We now estimate $\alpha$.

For $x_{0} \in B_{2 \delta}$ let $R=\tilde{c} r_{0}$ with $\tilde{c}$ sufficiently small and $B(y)=1\left(\left|y-x_{0}\right|<R\right)$. Omitting the $t$-dependence,

$$
|\alpha|\left(x_{0}\right) \leq \int_{\mathbb{R}^{3}} \frac{|J q(y)|}{\left|x_{0}-y\right|^{2}} d y \leq \int_{\mathbb{R}^{3}} \frac{|q(y)|}{\left|x_{0}-y\right|^{2}} B(y) d y+\int_{\mathbb{R}^{3}} \frac{|J q(y)|}{\left|x_{0}-y\right|^{2}}(1-B(y)) d y .
$$

From the Hölder inequality and (2.19), the first term on the right hand side is bounded by

$$
\int_{\mathbb{R}^{3}} \frac{|q(y)|}{\left|x_{0}-y\right|^{2}} B(y) d y \leq R^{-1+\varepsilon / 2}|t|^{-\varepsilon / 4} .
$$

From the Hölder inequality and (2.15), we have the following variation of (2.15) for $|x|<8 \delta$

$$
r_{x}^{-3} \int\left|1\left(|x-y| \leq r_{x} / 200\right) q(y, t)\right| d y \leq r_{x}^{-2+\varepsilon}|t|^{-\varepsilon / 2} .
$$

Multiply by

$$
\left|x_{0}-x\right|^{-2} \cdot 1\left(\left|x_{0}-x\right| \geq R / 40\right) \cdot 1(|x|<8 \delta),
$$

and integrate over $x$ to have

$$
\begin{gathered}
\iint r_{x}^{-3} \frac{1\left(|x-y| \leq r_{x} / 200\right)|q(y)| 1\left(\left|x_{0}-x\right| \geq R / 40\right)}{\left|x_{0}-x\right|^{2}} 1(|x|<8 \delta) d x d y, \\
\leq \int d x \frac{r_{x}^{-2+\varepsilon} t^{-\varepsilon / 2} 1\left(\left|x_{0}-x\right| \geq R / 40\right)}{\left|x_{0}-x\right|^{2}} \leq C R^{-1+\varepsilon}|t|^{-\varepsilon / 2} .
\end{gathered}
$$

The left hand side is bounded below by

$$
\begin{gathered}
\int r_{x}^{-3} \frac{1\left(|x-y| \leq r_{x} / 200\right)|q(y)| 1\left(\left|x_{0}-x\right| \geq R / 40\right)}{\left|x_{0}-x\right|^{2}} 1(|x|<8 \delta) d x d y \\
\geq C \int d x d y r_{y}^{-3} 1\left(|x-y| \leq r_{y} / 400\right)|J q|(y) \frac{(1-B(y))}{\left|x_{0}-y\right|^{2}} \geq C \int d y|J q|(y) \frac{(1-B(y))}{\left|x_{0}-y\right|^{2}} .
\end{gathered}
$$

Above for the first inequality we have used that $y$ is in a small neighborhood of $x$ for the integrand to be nonzero, in particular $r_{x} \sim r_{y}$ and $\left|x_{0}-x\right| \sim\left|x_{0}-y\right|$. We have thus proved that

$$
\int\left|x_{0}-y\right|^{-2}(1-B(y))|J q(y)| d y \leq C R^{-1+\varepsilon}|t|^{-\varepsilon / 2} .
$$

Since all $\varepsilon>0$ in the proofs are arbitrarily small, this proves the same bound for $\left|\alpha\left(x_{0}, t\right)\right|$. It follows that $\|\alpha(t)\|_{L_{x}^{1}\left(B_{2 \delta}\right)} \leq \int_{B_{2 \delta}} r_{x}^{-1+\varepsilon} t^{-\varepsilon / 2} d x \leq C t^{-\varepsilon / 2}$ and we get the pointwise bound for $b$ in $Q_{\delta}$. 


\section{The case $|v| \leq C r^{-1+\varepsilon}|t|^{-\varepsilon / 2}$}

In this section, we prove the estimate (1.5) from the assumption $|v| \leq C r^{-1+\varepsilon}|t|^{-\varepsilon / 2}$. Our main idea is the following Theorem 3.1 which states that the space singularity can be replaced by the time singularity.

Theorem 3.1 Suppose for some $\varepsilon \in(0,1 / 2)$ we have

$$
|v(x,-t)| \leq C r^{-1+\varepsilon} t^{-\varepsilon / 2}, \quad(x,-t) \in Q_{1} .
$$

Then for any $\delta \in(0,1)$ and $0<\alpha<1 / 2$, there is a constant $C$ such that

$$
|v(x,-t)| \leq C r^{-2 \alpha} t^{-1 / 2+\alpha}, \quad(x,-t) \in Q_{\delta} .
$$

Proof. We shall need the following Lemma which exchanges the space singularity with the time singularity by replacing $\varepsilon$ with $2 \varepsilon$. The idea of the proof is to view the Navier-Stokes equation as a linear equation with a source term $v \cdot \nabla v$. Since this term is in the form $v^{2}$, we naturally increase the time singularity to $|t|^{-\varepsilon}$. The spatial singularity will come out correctly due to the scaling invariance of the Navier-Stokes equation. This can be seen easily if we pretend that the kernel of the linear Stokes equation is a heat kernel. The general case only involves a minor technicality to deal with the divergence free condition.

Lemma 3.2 Suppose (3.1) holds in $Q_{1}$. Then for any $\tau \in(0,1)$ there is a constant $C$ such that

$$
|v(x,-t)| \leq C r^{-1+2 \varepsilon} t^{-\varepsilon}, \quad(x,-t) \in Q_{\tau} .
$$

Note that (3.1), (3.2) and (3.3) are all invariant under the natural scaling of (N-S). Assuming this Lemma, we now finish the proof of Theorem 3.1.

Suppose that (3.1) holds for some $\varepsilon \in(0,1 / 2)$. Then from Lemma 3.2, we can increase $\varepsilon$ by a factor of two. In fact, (3.1) and (3.3) implies that

$$
|v(x,-t)| \leq C r^{-1+\beta} t^{-\beta / 2}, \quad(x,-t) \in Q_{\tau},
$$

for all $\varepsilon \leq \beta \leq 2 \varepsilon$. Iterating this procedure, we obtained that (3.2) holds for $0<\alpha<(1-\varepsilon) / 2$. It remains to show that $(1-\varepsilon) / 2$ can be replaced with $1 / 2$.

We have shown that (3.2) holds for small $\alpha$. Notice that for small $\alpha$ condition (3.2) is very close to the assumption (1.2) (which is the case $\alpha=0$ ). One can easily check that all arguments in Section 2 remain valid if the assumption (1.2) is replaced by (3.2) if $\alpha$ is sufficiently small. Then the conclusion of Lemma 2.2 holds in this case. So that we are able to conclude that (3.1) holds for arbitrarily small $\varepsilon$. Iterating this procedure proves that (3.2) holds for $0<\alpha<1 / 2$.

To prove the lemma, we write the Navier-Stokes equations (N-S) as a Stokes system with force

$$
\partial_{t} v_{i}-\Delta v_{i}+\partial_{i} p=\partial_{j} f_{i j}, \quad f_{i j}=-v_{i} v_{j} .
$$

Recall key steps in [3]: $v=u+\tilde{v}$ where $\tilde{v}$ is defined as follows: Let $P$ be the Helmholtz projection in $\mathbb{R}^{3}$, i.e., $(P g)_{i}=g_{i}-R_{i} R_{k} g_{k}$. Let $\zeta(x, t) \in C^{\infty}\left(\mathbb{R}^{4}\right), \zeta \geq 0, \zeta=1$ on $Q_{\frac{1+\tau}{2}}$ and $\zeta=0$ on $\mathbb{R}^{3} \times(-\infty, 0]-Q_{1}$. Notice that we cutoff at order one. For a fixed $i$, define

$$
\tilde{v}_{i}(x, t)=\int_{-1}^{t} \Gamma(x-y, t-s) \partial_{j}\left(F_{i j}\right)(y, s) d y d s,
$$


where $\Gamma$ is the heat kernel and $F_{i j}=f_{i j} \zeta-R_{i} R_{k}\left(f_{k j} \zeta\right)$.

With this choice of $\tilde{v}, u$ satisfies the homogeneous Stokes system in $Q_{\frac{1+\tau}{2}}$ and the following bounds:

$$
\|\nabla u\|_{L_{t}^{s} L_{x}^{q}\left(Q_{\tau}\right)} \leq c\|u\|_{L_{t}^{s} L_{x}^{1}\left(Q_{1}\right)} \leq c\|v\|_{L_{t}^{s} L_{x}^{1}\left(Q_{1}\right)}+c\|\tilde{v}\|_{L_{t}^{s} L_{x}^{1}\left(Q_{1}\right)},
$$

provided that $1<s, q<\infty$. One can check that the proof in [3] gives (3.5) for $s=\infty$. The requirement $s<\infty$ is for the estimates of $\tilde{v}$.

Lemma 3.3 Under the assumption (3.1), we have

$$
\left|\tilde{v}\left(x_{0},-t\right)\right| \leq C r_{0}^{-1+2 \varepsilon} t^{-\varepsilon}, \quad\left(x_{0}, t\right) \in \mathbb{R}^{3} \times(0,1) .
$$

Proof. Denote $R=r_{0}$. Notice that assumption (3.1) implies (3.3) when $R \geq \sqrt{t}$. Hence we may assume that $R \leq \sqrt{t}$. Let $h=f_{i j} \zeta$ and $K=R_{i} R_{j}$. Denote

$$
\begin{aligned}
\xi_{1}(x,-t) & =\int_{-1}^{-t} \int|s|^{-2} \exp \left[-\frac{|x-\tilde{x}|^{2}}{4(-t-s)}\right] h(\tilde{x}, s) d \tilde{x} d s \\
& =\int_{0}^{1-t} \int s^{-2} \exp \left[-\frac{|x-\tilde{x}|^{2}}{4 s}\right] h(\tilde{x},-t-s) d \tilde{x} d s, \\
\xi_{2}(x,-t) & =\int_{0}^{1-t} \int s^{-2} \exp \left[-\frac{|x-\tilde{x}|^{2}}{4 s}\right] K h(\tilde{x},-t-s) d \tilde{x} d s .
\end{aligned}
$$

We can bound $\tilde{v}(x,-t)$ pointwisely by $\sum_{i, j, k}\left|\xi_{k}(x,-t)\right|$. Thus it suffices to show $\sum\left|\xi_{k}\left(x_{0}, t\right)\right| \leq C R^{-1+2 \varepsilon} t^{-\varepsilon}$. We shall only bound $\xi_{2}$ since the bound for $\xi_{1}$ is identical. For $x_{0}$ fixed, let

$$
g(x, s)=\exp \left[-\frac{\left|x_{0}-x\right|^{2}}{4 s}\right] s^{-3 / 2}
$$

Since $K$ is symmetric, we have

$$
\xi_{2}\left(x_{0},-t\right)=\int_{0}^{1-t} s^{-1 / 2} \int d x(K g)(x, s) h(x,-t-s) d s .
$$

Let $h_{1}(x, a)=1(r \geq R) h(x, a)$ and $h_{2}(x, a)=1(r \leq R) h(x, a)$. Then we have

$$
\int d x(K g)(x, s) h_{1}(x,-t-s) \leq\left[\int d x(K g)^{p}(x, s)\left|x-x_{0}\right|^{p}\right]^{1 / p}\left[\int d x h_{1}^{q}(x,-t-s)\left|x-x_{0}\right|^{-q}\right]^{1 / q} .
$$

Recall $|x|^{a}$ is an $A_{p}$ weight in $\mathbb{R}^{n}$ provided that

$$
-n<a<n(p-1) .
$$

Thus for

$$
0 \leq p<3 p-3
$$

we have

$$
\left[\int d x(K g)^{p}(x, s)\left|x-x_{0}\right|^{p}\right]^{1 / p} \leq\left[\int d x g^{p}(x, s)\left|x-x_{0}\right|^{p}\right]^{1 / p} \leq|s|^{-1+3 /(2 p)}=|s|^{-3 /(2 q)+1 / 2} .
$$


Since $h_{1}$ is supported in $r \geq R$, we have for

$$
(3-2 \varepsilon) q>3, \quad q>1,
$$

the following inequality:

$$
\left[\int d x h_{1}^{q}(x,-t-s)\left|x-x_{0}\right|^{-q}\right]^{1 / q} \leq t^{-\varepsilon}\left[\int_{r \geq R} d x r^{(-2+2 \varepsilon) q}\left|x-x_{0}\right|^{-q}\right]^{1 / q} \leq t^{-\varepsilon} R^{-3+2 \varepsilon+3 / q},
$$

where we have used (3.1) in the first inequality. Thus we have

$$
s^{-1 / 2} \int d x(K g)(x, s) h_{1}(x,-t-s) \leq t^{-\varepsilon} R^{-3+2 \varepsilon}\left(\frac{R^{2}}{s}\right)^{3 /(2 q)} .
$$

Therefore, we have

$$
\int_{0}^{R^{2}} s^{-1 / 2} \int d x(K g)(x, s) h_{1}(x,-t-s) d s \leq C t^{-\varepsilon} R^{-3+2 \varepsilon} \int_{0}^{R^{2}} d s\left(\frac{R^{2}}{s}\right)^{3 /(2 q)} \leq C t^{-\varepsilon} R^{-(1-2 \varepsilon)}
$$

provided that

$$
3 /(2 q)<1, \quad(3-2 \varepsilon) q>3, \quad q>1, \quad 0 \leq p<3 p-3 .
$$

For any $0<\varepsilon<1 / 2$ fixed, we can solve the last condition by

$$
q=(3 / 2)^{+} .
$$

Similarly,

$$
\int_{R^{2}}^{1} s^{-1 / 2} \int d x(K g)(x, s) h_{1}(x,-t-s) d s \leq C t^{-\varepsilon} R^{-3+2 \varepsilon} \int_{R^{2}}^{1} d s\left(\frac{R^{2}}{s}\right)^{3 /(2 q)} \leq C t^{-\varepsilon} R^{-(1-2 \varepsilon)},
$$

provided that

$$
3 /(2 q)>1, \quad(3-2 \varepsilon) q>3, \quad q>1, \quad 0 \leq p<3 p-3 .
$$

For any $0<\varepsilon<1 / 2$ fixed, we can solve the last condition by

$$
q=(3 / 2)^{-} .
$$

For any $m>0$ and $a, b$ dual we have

$$
\int d x(K g)(x, s) h_{2}(x, s) \leq\left[\int d x(K g)^{a}(x, s)\left|x-x_{0}\right|^{m a}\right]^{1 / a}\left[\int d x h_{2}^{b}(x,-t-s)\left|x-x_{0}\right|^{-m b}\right]^{1 / b} .
$$

If

$$
m a<3 a-3,
$$

then $|x|^{m a}$ is an $A_{a}$ weight. Thus we have

$$
\left[\int d x(K g)^{a}(x, s)\left|x-x_{0}\right|^{m a}\right]^{1 / a} \leq\left[\int d x g^{a}(x, s)\left|x-x_{0}\right|^{m a}\right]^{1 / a} \leq s^{-3 /(2 b)+m / 2} .
$$


We can estimate the last integral by

$$
\begin{gathered}
\int d x h_{2}^{b}(x,-t-s)\left|x-x_{0}\right|^{-m b} \leq t^{-\varepsilon b} \int d x r^{-(2-2 \varepsilon) b} 1(r \leq R)\left|x-x_{0}\right|^{-m b} \\
=t^{-\varepsilon b} R^{-b(2+m-2 \varepsilon)+3} \int d x r^{-(2-2 \varepsilon) b} 1(r \leq 1)\left|x-\left(x_{0} / R\right)\right|^{-m b} \leq C t^{-\varepsilon b} R^{-b(2+m-2 \varepsilon)+3},
\end{gathered}
$$

where the equality is due to scaling and we have assumed that

$$
(1-\varepsilon) b<1, \quad 1<m b<3 .
$$

Therefore, we have

$$
\int_{0}^{R^{2}} s^{-1 / 2} \int d x(K g)(x, s) h_{2}(x,-t-s) d s \leq C t^{-\varepsilon} R^{-(3-2 \varepsilon)} \int_{0}^{R^{2}} d s\left(\frac{R^{2}}{s}\right)^{3 /(2 b)-m / 2+1 / 2} \leq C t^{-\varepsilon} R^{-(1-2 \varepsilon)},
$$

provided that

$$
b>\frac{3}{m+1}, \quad(1-\varepsilon) b<1, \quad 1<m b<3, \quad m a<3 a-3 .
$$

Since $a$ and $b$ are dual, the last condition is equivalent to $m<3 / b$, which is part of the third condition. It is easy to check that the following condition implies (3.11)

$$
1 / 2<m, \quad \frac{3}{m+1}<b<\frac{3}{m}, \quad b<\frac{1}{1-\varepsilon} .
$$

For any $0<\varepsilon<1 / 2$ fixed, we can solve the last condition by

$$
b=\frac{1}{1-\varepsilon}-\mu, \quad m=3-3 \varepsilon,
$$

for $\mu$ small enough.

Similarly, we have

$$
\begin{gathered}
\int_{R^{2}}^{1} s^{-1 / 2} \int d x(K g)(x, s) h_{2}(x,-t-s) d s \leq C t^{-\varepsilon} R^{-(3-2 \varepsilon)} \int_{R^{2}}^{1} d s\left(\frac{R^{2}}{s}\right)^{3 /(2 b)-m / 2+1 / 2} \leq C t^{-\varepsilon} R^{-(1-2 \varepsilon)} \\
b<\frac{3}{m+1}, \quad(1-\varepsilon) b<1, \quad 1<m b<3, \quad m a<3 a-3
\end{gathered}
$$

It is easy to check that the following condition implies (3.14)

$$
\frac{1}{m}<b<\frac{3}{m+1}, \quad b<\frac{1}{1-\varepsilon} .
$$

This equation has a solution provided that there is an $m$ solving the equation

$$
1-\varepsilon<m<2-3 \varepsilon .
$$

This is clearly so for any $0<\varepsilon<1 / 2$ fixed. We have thus proved the Lemma. 
We now conclude the proof of Lemma 3.2. Let $\gamma=\frac{1-\tau}{4}$ and denote $Q_{1}^{t}=B_{1}(0) \times(-1,-t)$. For any $\left(x_{0}, t\right) \in Q_{\tau}, Q_{2 \gamma}\left(x_{0}, t\right) \subset Q_{\frac{1+\tau}{2}} \cap Q_{1}^{t}$ and hence $u$ satisfies the homogeneous Stokes system in $Q_{2 \gamma}\left(x_{0}, t\right)$. By (3.5), assumption (3.1) and Lemma 3.3, we have

$$
\|\nabla u\|_{L_{t}^{\infty} L_{x}^{4}\left(Q_{\gamma}\left(x_{0}, t\right)\right)} \leq c\|u\|_{L_{t}^{\infty} L_{x}^{1}\left(Q_{2 \gamma}\left(x_{0}, t\right)\right)} \leq c\|v\|_{L_{t}^{\infty} L_{x}^{1}\left(Q_{1}^{t}\right)}+c\|\tilde{v}\|_{L_{t}^{\infty} L_{x}^{1}\left(Q_{1}^{t}\right)} \leq c t^{-\varepsilon / 2}+c t^{-\varepsilon} \leq c t^{-\varepsilon} .
$$

By the Sobolev inequality,

$$
\|u\|_{L_{t}^{\infty} L_{x}^{\infty}\left(Q_{\gamma}\left(x_{0}, t\right)\right)} \leq c\|\nabla u\|_{L_{t}^{\infty} L_{x}^{4}\left(Q_{\gamma}\left(x_{0}, t\right)\right)}+c\|u\|_{L_{t}^{\infty} L_{x}^{1}\left(Q_{2 \gamma}\left(x_{0}, t\right)\right)} \leq c t^{-\varepsilon} .
$$

Together with (3.6), we have thus proved Lemma 3.2.

\section{Local Maximum Estimate}

In sections 2 and 3 we have proved the bound (1.5) under both assumptions (1.2) and (1.3) with $\varepsilon>0$. Our goal in the remaining sections 4,5 , and 6 is to show that the proof in the paper [3] can be extended in this case. This section proves local maximum estimates assuming (1.5). These estimates will be used to obtain Hölder continuity of $r v_{\theta}$ in section 5 and to bound $\Omega=\bar{\omega}_{\theta} / r$ of the limit solution in section 6 .

Suppose $u$ is the smooth function satisfying

$$
\partial_{t} u-L^{*} u=0, \quad L=\Delta+\frac{2}{r} \partial_{r}-b \cdot \nabla,
$$

We now derive parabolic De Giorgi type energy estimates for this equation under the assumption

$$
|b| \leq C_{*} r^{-1+2 \varepsilon}|t|^{-\varepsilon} .
$$

Above $C_{*}>0$ is an absolute constant which is allowed to be large, above $\epsilon>0$ is sufficiently small.

Consider a test function $0 \leq \zeta_{1}(x, t) \leq 1$ defined on $Q_{1}$ for which $\zeta_{1}=0$ on $\partial B_{1} \times\left[-1^{2}, 0\right]$ and $\zeta_{1}=1$ on $Q_{\sigma}$ for $0<\sigma<1$. Suppose that $\zeta_{1}(x,-1)=0$. Now consider the rescaled test function $\zeta(x, t)=\zeta_{1}\left(x / R, t / R^{2}\right)$ on $Q_{R}$. Define $(u)_{ \pm}=\max \{ \pm u, 0\}$ for a scalar function $u$. Multiply $(4.1)$ by $p(u-k)_{ \pm}^{p-1} \zeta^{2}$ for $1<p \leq 2$ and $k \geq 0$ to obtain

$$
\begin{gathered}
\left.\int_{B_{R}} \zeta^{2}(u-k)_{ \pm}^{p}\right|_{-R^{2}} ^{t}+\frac{4(p-1)}{p} \int_{-R^{2}}^{t} d t^{\prime} \int_{B_{R}} d x\left|\nabla\left((u-k)_{ \pm}^{p / 2} \zeta\right)\right|^{2} \\
=2 \int_{-R^{2}}^{t} d t^{\prime} \int_{B_{R}} d x(u-k)_{ \pm}^{p}\left(\zeta \frac{\partial \zeta}{\partial t}+|\nabla \zeta|^{2}+\frac{2-p}{p} \zeta \Delta \zeta-2 \zeta \frac{\partial_{r} \zeta}{r}+\bar{b} \cdot \zeta \nabla \zeta\right) \\
-\left.2 \int_{-R^{2}}^{t} d t^{\prime} \int_{B_{R}} d z \zeta^{2}(u-k)_{ \pm}^{p}\right|_{r=0} .
\end{gathered}
$$

Notice that the last term is negative.

Let $v_{ \pm} \equiv(u-k)_{ \pm}^{p / 2}$. To estimate the term involving $b$ we use Young's inequality

$$
\int_{\mathbb{R}^{3}} v_{ \pm}^{2} b \zeta \cdot \nabla \zeta \leq \delta \frac{R^{-1+\alpha}}{1+\alpha} \int_{\mathbb{R}^{3}} v_{ \pm}^{2} \zeta^{2}\left|b\left(\frac{t}{R^{2}}\right)^{\varepsilon}\right|^{1+\alpha}+C_{\delta} \frac{\alpha R^{-2+(1+\alpha) / \alpha}}{1+\alpha} \int_{\mathbb{R}^{3}} v_{ \pm}^{2} \zeta^{2}\left[\left(\frac{R^{2}}{t}\right)^{\varepsilon} \frac{|\nabla \zeta|}{\zeta}\right]^{(1+\alpha) / \alpha}
$$


This holds for small $\delta>0$ and $\alpha>0$ to be chosen. Further choose $\zeta$ to decay like $(1-|x| / R)^{n}$ near the boundary of $B_{R}$. If $n$ is large enough (depending on $\alpha$ ) we have

$$
C_{\delta} \frac{\alpha R^{-2+(1+\alpha) / \alpha}}{1+\alpha} \int_{\mathbb{R}^{3}} v_{ \pm}^{2} \zeta^{2}\left[\left(\frac{R^{2}}{t}\right)^{\varepsilon} \frac{|\nabla \zeta|}{\zeta}\right]^{(1+\alpha) / \alpha} \leq C R^{-2}\left(\frac{R^{2}}{t}\right)^{\varepsilon(1+\alpha) / \alpha} \int_{B_{R}} v_{ \pm}^{2}
$$

We also use the Hölder and Sobolev inequalities to obtain

$$
\begin{aligned}
\delta \frac{R^{-1+\alpha}}{1+\alpha} \int_{\mathbb{R}^{3}} v_{ \pm}^{2} \zeta^{2}\left|b\left(\frac{t}{R^{2}}\right)^{\varepsilon}\right|^{1+\alpha} & \leq \delta\left(R^{(-1+\alpha) 3 / 2} \int_{B_{R}}\left|b\left(\frac{t}{R^{2}}\right)^{\varepsilon}\right|^{(1+\alpha) 3 / 2}\right)^{2 / 3} \int_{\mathbb{R}^{3}}\left|\nabla\left(v_{ \pm} \zeta\right)\right|^{2} \\
& \leq \delta C \int_{\mathbb{R}^{3}}\left|\nabla\left(v_{ \pm} \zeta\right)\right|^{2}
\end{aligned}
$$

For $b$ satisfies (4.2), there is an $\alpha$ small enough so that the last inequality holds. We conclude that

$$
\int_{\mathbb{R}^{3}} v_{ \pm}^{2} b \zeta \cdot \nabla \zeta \leq \delta C \int_{\mathbb{R}^{3}}\left|\nabla\left(v_{ \pm} \zeta\right)\right|^{2}+C R^{-2} \int_{B_{R}}\left(\frac{R^{2}}{t}\right)^{\varepsilon(1+\alpha) / \alpha} v_{ \pm}^{2} .
$$

We have $\partial_{r} \zeta / r=\partial_{\rho} \zeta / \rho$ since $\zeta$ is radial; so that the singularity $1 / \rho$ is effectively $1 / R$. We thus have

$$
\begin{aligned}
\sup _{-\sigma^{2} R^{2}<t<0} \int_{B_{\sigma R} \times\{t\}}\left|(u-k)_{ \pm}\right|^{p} & +\int_{Q_{\sigma R}}\left|\nabla(u-k)_{ \pm}^{p / 2}\right|^{2} \\
& \leq \frac{C_{*}}{(1-\sigma)^{2} R^{2}} \int_{Q_{R}}\left(\frac{R^{2}}{t}\right)^{\varepsilon(1+\alpha) / \alpha}\left|(u-k)_{ \pm}\right|^{p} .
\end{aligned}
$$

Our goal will be to establish $L^{p}$ to $L^{\infty}$ bounds for functions in this energy class.

The estimates in this section will be proven for a general function $u=\Omega$ satisfying (4.4):

Lemma 4.1 Suppose u satisfies (4.4) for $1<p \leq 2$ with $\epsilon>0$ sufficiently small. Then for $0<R \leq 1$ we have the estimate

$$
\sup _{Q_{R / 2}} u_{ \pm} \leq C\left(p, C_{*}\right)\left(R^{-3-2} \int_{Q_{R}}\left|u_{ \pm}\right|^{p}\right)^{1 / p} .
$$

Proof. For $K>0$ to be determined and $N$ a positive integer we define

$$
\begin{gathered}
k_{N}=k_{N}^{ \pm}=\left(1 \mp 2^{-N}\right) K, R_{N}=\left(1+2^{-N}\right) R / 2, \rho_{N}=\frac{R}{2^{N+3}}, \\
R_{N+1}<\bar{R}_{N}=\left(R_{N}+R_{N+1}\right) / 2<R_{N} .
\end{gathered}
$$

Notice that

$$
R_{N}-\bar{R}_{N}=\left(R_{N}-R_{N+1}\right) / 2=\left(2^{-N}-2^{-N-1}\right) R / 4=\rho_{N} .
$$

Define $Q_{N}=Q\left(R_{N}\right)$ and $\bar{Q}_{N}=Q\left(\bar{R}_{N}\right) \subset Q_{N}$. Choose a smooth test function $\zeta_{N}$ satisfying $\zeta_{N} \equiv 1$ on $\bar{Q}_{N}$, $\zeta \equiv 0$ outside $Q_{N}$ and vanishing on it's spatial boundary, $0 \leq \zeta_{N} \leq 1$ and $\left|\nabla \zeta_{N}\right| \leq \rho_{N}^{-1}$ in $Q_{N}$. Further let

$$
A^{ \pm}(N)=\left\{X \in Q_{N}: \pm\left(u-k_{N+1}\right)(X)>0\right\} .
$$


And $A_{N, \pm}=\left|A^{ \pm}(N)\right|$. Let $v_{ \pm}=\zeta_{N}\left(u-k_{N+1}\right)_{ \pm}^{p / 2}$.

Let $\gamma$ be a positive constant (to be chosen) such that $\gamma-1>0$ is very small. Hölder's inequality yields

$$
\begin{aligned}
\int_{Q_{N+1}}\left|\left(u-k_{N+1}\right)_{ \pm}\right|^{p} & \leq \int_{\bar{Q}_{N}}\left|v_{ \pm}\right|^{2} \\
& \leq\left(\int_{\bar{Q}_{N}}\left|v_{ \pm}\right|^{2(n+2) /(n \gamma)}\right)^{n \gamma /(n+2)} A_{N, \pm}^{(2+n(1-\gamma)) /(n+2)} .
\end{aligned}
$$

We will use the following Sobolev inequality which holds for functions vanishing on $\partial B_{R}$ :

$$
\int_{Q_{R}}|u|^{2(n+2) / n} \leq C(n)\left(\sup _{-R^{2}<t<0} \int_{B_{R} \times\{t\}}|u|^{2}\right)^{2 / n} \int_{Q_{R}}|\nabla u|^{2} .
$$

See $[9$, Theorem 6.11, p.112]. Above and below $n$ is the spatial dimension, so that $n=3$.

Since $v_{ \pm}$vanishes on the spatial boundary of $Q_{N}$ we have

$$
\begin{aligned}
& \left(\int_{\bar{Q}_{N}}\left|v_{ \pm}\right|^{2(n+2) /(n \gamma)}\right)^{n \gamma /(n+2)} \leq\left(\int_{Q_{N}}\left|v_{ \pm}\right|^{2(n+2) /(n \gamma)}\right)^{n \gamma /(n+2)} \\
\leq & {\left[\left(\sup _{-R_{N}^{2}<t<0} \int_{B\left(R_{N}\right) \times\{t\}}\left|v_{ \pm}\right|^{2 / \gamma}\right)^{2 /(n+2)}\left(\int_{Q_{N}}\left|\nabla\left(v_{ \pm}\right)^{1 / \gamma}\right|^{2}\right)^{n /(n+2)}\right]^{\gamma} . }
\end{aligned}
$$

We use Young's inequality to bound this above by

$$
\leq C\left[\left(\sup _{-R_{N}^{2}<t<0} \int_{B\left(R_{N}\right) \times\{t\}}\left|v_{ \pm}\right|^{2 / \gamma}+\int_{Q_{N}}\left|\nabla\left(v_{ \pm}\right)^{1 / \gamma}\right|^{2}\right)\right]^{\gamma} .
$$

From (4.4) the above is bounded as

$$
\begin{gathered}
\leq C\left[\left(\sup _{-R_{N}^{2}<t<0} \int_{B\left(R_{N}\right) \times\{t\}}\left|\left(u-k_{N+1}\right)_{ \pm}\right|^{p / \gamma}+\int_{Q_{N}}\left|\nabla\left(u-k_{N+1}\right)_{ \pm}^{p /(2 \gamma)}\right|^{2}\right)+\frac{C}{\rho_{N}^{2}} \int_{Q_{N}}\left|\left(u-k_{N+1}\right)_{ \pm}\right|^{p / \gamma}\right]^{\gamma} \\
\leq\left[\frac{C}{\rho_{N}^{2}} \int_{Q_{N}}\left[1+\left(\frac{R^{2}}{t}\right)^{\varepsilon(1+\alpha) / \alpha}\right]\left|\left(u-k_{N+1}\right)_{ \pm}\right|^{p / \gamma}\right]^{\gamma} \\
\leq\left\{\frac{C}{\rho_{N}^{2}} \int_{Q_{N}}\left|\left(u-k_{N}\right)_{ \pm}\right|^{p}\right\}\left\{\frac{C}{\rho_{N}^{2}} \int_{Q_{N}}\left[1+\left(\frac{R^{2}}{t}\right)^{\varepsilon(1+\alpha) / \alpha}\right]^{\beta}\right\}^{\gamma / \beta} .
\end{gathered}
$$

Above $\beta$ is the dual exponent of $\gamma$, i.e. $\frac{1}{\gamma}+\frac{1}{\beta}=1$. For the upper bound above to be finite we require that $\varepsilon<\alpha /[\beta(1+\alpha)]$. Since $\beta \geq 1$ we have

$$
\int_{Q_{N}}\left[1+\left(\frac{R^{2}}{t}\right)^{\varepsilon(1+\alpha) / \alpha}\right]^{\beta} \leq C \int_{Q_{N}}\left[1+\left(\frac{R^{2}}{t}\right)^{\varepsilon(1+\alpha) \beta / \alpha}\right] \leq C R_{N}^{5} .
$$


From here our next upper bound is

$$
\leq C\left(\frac{R_{N}^{5}}{\rho_{N}^{2}}\right)^{\gamma / \beta}\left\{\frac{1}{\rho_{N}^{2}} \int_{Q_{N}}\left|\left(u-k_{N}\right)_{ \pm}\right|^{p}\right\} \leq C\left(16 R^{3} 2^{2 N}\right)^{\gamma / \beta}\left\{\frac{1}{\rho_{N}^{2}} \int_{Q_{N}}\left|\left(u-k_{N}\right)_{ \pm}\right|^{p}\right\} .
$$

Further assume $K^{p} \geq R^{-n-2} \int_{Q(R)}\left|u_{ \pm}\right|^{p}$. Now define

$$
Y_{N} \equiv K^{-p} R^{-n-2} \int_{Q_{N}}\left|\left(u-k_{N}\right)_{ \pm}\right|^{p} .
$$

Since $k_{N}^{ \pm}$are increasing for + or decreasing for - and $Q_{N}$ are decreasing, $Y_{N}$ is decreasing.

Chebyshev's inequality tells us that

$$
\begin{gathered}
A_{N, \pm}=\left|\left\{Q_{N}: \pm\left(u-k_{N+1}^{ \pm}\right)>0\right\}\right|=\left|\left\{Q_{N}: \pm\left(u-k_{N}^{ \pm}\right)> \pm\left(k_{N+1}^{ \pm}-k_{N}^{ \pm}\right)\right\}\right| \\
=\left|\left\{Q_{N}: \pm\left(u-k_{N}\right)>K / 2^{N+1}\right\}\right| \leq 2^{p(N+1)} R^{n+2} Y_{N} .
\end{gathered}
$$

Putting all of this together yields

$$
\begin{gathered}
\int_{Q_{N+1}}\left|\left(u-k_{N+1}\right)_{ \pm}\right|^{p} \leq\left(\int_{\bar{Q}_{N}}\left|v_{ \pm}\right|^{2(n+2) /(n \gamma)}\right)^{n \gamma /(n+2)} A_{N, \pm}^{(2+n(1-\gamma)) /(n+2)} \\
\leq C\left(\frac{1}{\rho_{N}^{2}} \int_{Q_{N}}\left|\left(u-k_{N}\right)_{ \pm}\right|^{p}\right) R^{3 \gamma / \beta} 2^{2 N \gamma / \beta}\left(2^{p(N+1)} R^{n+2} Y_{N}\right)^{(2+n(1-\gamma)) /(n+2)} \\
\leq C\left(\frac{1}{\rho_{N}^{2}} K^{p} R^{n+2} Y_{N}\right) R^{3 \gamma / \beta} 2^{2 N \gamma / \beta}\left(2^{p(N+1)} R^{n+2} Y_{N}\right)^{(2+n(1-\gamma)) /(n+2)} \\
\leq C\left(C_{*} n\right) K^{p} R^{n+2} 2^{q N} Y_{N}^{1+(2+n(1-\gamma)) /(n+2)} .
\end{gathered}
$$

We have just used $R \leq 1$. Also the exponent is given by

$$
q=\frac{[2+n(1-\gamma)] p}{n+2}+\frac{2 \gamma}{\beta}+2
$$

We have thus shown

$$
Y_{N+1} \leq C\left(C_{*}, n\right) 2^{q N} Y_{N}^{1+(2+n(1-\gamma)) /(n+2)} .
$$

We now choose $\gamma>1$ such that the exponent of $Y_{N}$ is larger than one: $2+n(1-\gamma)>0$.

One can check that if $\kappa$ is large enough, then the following identity will be preserved by (4.5):

$$
Y_{N} \leq 2^{-\kappa N}
$$

We are still free to choose $K$ large enough such that the following initial condition holds: $Y_{1} \leq 2^{-\kappa}$.

\section{$5 \quad$ Hölder Continuity}

In this section we prove Hölder continuity of the function $\Gamma=r v_{\theta}$ at $t=0$ under the assumption (1.5). Earlier than $t=0$, the function $\Gamma$ is smooth. Additionally $\Gamma$ satisfies

$$
\frac{d \Gamma}{d t}-L \Gamma=0, \quad L=\Delta-\frac{2}{r} \frac{\partial}{\partial r}-b \cdot \nabla .
$$


Notice that $\Gamma(r=0, t)=0$ for all $-1 \leq t<0$. One can check, using this condition, that both (4.4) and Lemma 4.1 hold. Together with (4.2) we then have

$$
\sup _{-1 \leq t<0}\|\Gamma(t)\|_{L^{\infty}\left(B_{R}\right)} \leq C<\infty .
$$

Our argument makes use Nash's fundamental idea for a lower bound (Lemma 5.2). We consider this interesting in particular because the lower bound is obtained for a solution directly rather than the usual lower bound for a fundamental solution.

\subsection{Preliminary Bounds}

Let $X=(x, t)$. Define the modified parabolic cylinder at the origin

$$
Q(R, \tau)=\left\{X:|x|<R,-\tau R^{2}<t<0\right\} .
$$

Here $R>0$ and $\tau \in(0,1]$. We sometimes for brevity write $Q_{R}=Q(R)=Q(R, 1)$. Let

$$
m_{2} \equiv \inf _{Q(2 R)} \Gamma, \quad M_{2} \equiv \sup _{Q(2 R)} \Gamma, \quad M \equiv M_{2}-m_{2}>0 .
$$

Notice that $m_{2} \leq 0 \leq M_{2}$ since $\left.\Gamma\right|_{r=0}=0$.

Define

$$
u \equiv \begin{cases}2\left(\Gamma-m_{2}\right) / M & \text { if } \quad-m_{2}>M_{2}, \\ 2\left(M_{2}-\Gamma\right) / M & \text { else. }\end{cases}
$$

In either case

$$
0 \leq u(x, t) \leq 2,\left.\quad a \equiv u\right|_{r=0} \geq 1,
$$

and $u$ solves the equation (5.1).

Since now $u$ is nonnegative, we can make $u$ positive by adding arbitrary small constant to $u$. This part of argument is standard and from now on we assume that $u>0$.

\subsection{Lower bound on $\|u\|_{p}$}

Our goal in this section is to prove that there is a lower bound on $u$ with a more general assumption than that was used in our previous paper[3]. The bound that we prove in this section will serve as an input for Nash's argument as we shall describe it later on. Actually, we only need a lower bound on $\|u\|_{p}$ for some $0<p<1$.

Consider the following probability measure on $Q_{R}$

$$
d \omega=R^{-2} d t R^{-3} d x .
$$

Define the norm

$$
\|f\|_{L_{t, x}^{q, p}(\omega)}:=\left(\int_{-R^{2}}^{0} \frac{d t}{R^{2}}\left(\int_{B_{R}} \frac{d x}{R^{3}}|f|^{p}\right)^{q / p}\right)^{1 / q} .
$$

We will sometimes write $L^{p}(\omega)=L_{t, x}^{p, p}(\omega)$. Our main result in this section is the following 
Lemma 5.1 Suppose $u$ solves (5.1) and satisfies condition (5.3). Assume that for some small $\beta>1$ we have

$$
\|b\|_{L_{t, x}^{3 / 2, \beta}(\omega)} \leq C R^{-1} .
$$

Then for arbitrary $p \in(0,1)$ the following holds:

$$
a \leq C\|u\|_{L^{p}(\omega)}^{p / \alpha} .
$$

Above $\alpha$ is the dual exponent to $\beta$.

Notice that for $b$ satisfying (4.2) with any $0 \leq \epsilon \leq 1 / 2$ fixed, there is a $\beta$ such that the condition (5.5) is satisfied. The following proof is a small modification of the proof in [3].

Proof. We test the equation with $p u^{p-1} \zeta^{2}$ for $0<p<1$ and $\zeta \geq 0$ to have

$$
-I_{7}=\sum_{j=2}^{6} I_{j} .
$$

where

$$
\begin{aligned}
& \int_{Q(R)} p u^{p-1} \zeta^{2} \frac{\partial u}{\partial t}= {\left[\int_{B_{R}} \zeta^{2} u^{p}\right]_{t_{1}}^{0}-\int_{Q(R)} u^{p} 2 \zeta \frac{\partial \zeta}{\partial t} \equiv I_{1}+I_{2}, } \\
& \int_{Q(R)} p u^{p-1} \zeta^{2}(-\Delta u)= \frac{4(p-1)}{p} \int_{Q(R)}\left|\nabla\left(u^{p / 2} \zeta\right)\right|^{2} \\
&+\int_{Q(R)} 2 u^{p}\left[-|\nabla \zeta|^{2}+\frac{p-2}{p} \zeta \Delta \zeta\right] \equiv I_{3}+I_{4}, \\
& \int_{Q(R)} p u^{p-1} \zeta^{2} b \cdot \nabla u=-\int_{Q(R)} 2 u^{p} b \cdot \zeta \nabla \zeta \equiv I_{5}, \\
& \int_{Q(R)} p u^{p-1} \zeta^{2} \frac{2}{r} \partial_{r} u=-\int_{Q(R)} 4 u^{p} \zeta \zeta_{\rho} / \rho-\left.\int_{-R^{2}}^{0} d t \int_{\mathbb{R}} d z 2\left(\zeta^{2} u^{p}\right)\right|_{r=0} \\
& \equiv I_{6}+I_{7} .
\end{aligned}
$$

For arbitrary $p \in(0,1)$, we see that $I_{3}$ and $I_{7}$ are both non-positive.

Recall $\rho=|x|$. We choose $\zeta=\zeta_{1}(\rho) \zeta_{2}(t)$ where $\zeta_{1}(\rho)=1$ in $B_{R / 2}$ and $\zeta_{1}(\rho)$ has compact support in $B_{R}$; also $\zeta_{2}(t)=1$ if $t \in\left(-\frac{7}{8} R^{2},-\frac{1}{8} R^{2}\right)$ and $\zeta_{2}(t)$ has compact support in $\left(-R^{2}, 0\right)$. Thus $I_{1}=0$ and we have

$$
\frac{6}{4} R^{3} a^{p} \leq-I_{7}=\sum_{j=2}^{6} I_{j} .
$$

Clearly,

$$
I_{2} \leq \frac{C}{R^{2}} \int_{Q(R)} u^{p}, \quad I_{3} \leq 0, \quad I_{4}+I_{6} \leq \frac{C}{R^{2}} \int_{Q(R)} u^{p} .
$$

For any dual $\alpha, \beta$, we now bound $I_{5}$ :

$$
\left|I_{5}\right| \leq R^{5} R^{-1}\|b\|_{L_{t, x}^{3 / 2, \beta}(\omega)}\left\|u^{p}\right\|_{L_{t, x}^{3, \alpha}(\omega)} .
$$


We thus have for any $\alpha \geq 3$

$$
a^{p} \leq \frac{C}{R^{5}} \int_{Q(R)} u^{p}+\left\|u^{p}\right\|_{L_{t, x}^{3, \alpha}(\omega)} \leq\left\|u^{p}\right\|_{L_{t, x}^{\alpha}(\omega)} \leq\|u\|_{L^{\alpha p}(\omega)}^{p} .
$$

Since $p$ is arbitrary positive number less than one, this proves the Lemma. Notice that we only use $I_{3} \leq 0$ in this case.

\subsection{Nash inequality}

In this section we prove a Nash inequality. We shall need this inequality in the next section when we prove a Nash lower bound estimate.

Consider a function $f$, which satisfies the bounds $0 \leq f \leq M$ for some $M \geq 1$. Let $\mu$ be a probability measure. Now consider the average

$$
\alpha=\int \log f d \mu \text {. }
$$

And define $g=\log f-\alpha$. We have the following inequality

$$
\frac{\|f\|_{1}}{M}\left|\alpha-\log \int f d \mu\right| \leq\|g\|_{2} .
$$

Above we are using the following definition

$$
\|g\|_{p}:=\left(\int|g|^{p} d \mu\right)^{1 / p} .
$$

In the rest of this section we will give a short proof.

Proof. For $0 \leq \beta \leq 1$ we have

$$
\partial_{\beta} \log \int e^{\beta g} d \mu=\frac{\int g e^{\beta g} d \mu}{\int e^{\beta g} d \mu}=\frac{\int g f^{\beta} d \mu}{\int f^{\beta} d \mu} \leq \frac{\|g\|_{2}\left\|f^{\beta}\right\|_{2}}{\left\|f^{\beta}\right\|_{1}} \leq \frac{\|g\|_{2} M^{\beta}}{\left\|f^{\beta}\right\|_{1}} .
$$

Additionally, since $f$ is bounded, we have

$$
\int f^{\beta} d \mu=M^{\beta} \int\left(\frac{f}{M}\right)^{\beta} d \mu \geq M^{\beta} \int\left(\frac{f}{M}\right) d \mu=M^{\beta-1}\|f\|_{1} .
$$

We conclude

$$
\partial_{\beta} \log \int e^{\beta g} d \mu \leq \frac{M\left\|_{g}\right\|_{2}}{\|f\|_{1}} .
$$

This is all we need. Now integrate the above with respect to $\beta$ from 0 to 1 to obtain

$$
\log \int e^{g} d \mu=\log \int f d \mu-\alpha \leq \frac{M\|g\|_{2}}{\|f\|_{1}} .
$$

Hence we have (5.7). 


\subsection{Nash's lower bound}

Consider solutions $u$ to the equation (5.1) which satisfy (5.3). Let $v=-\log u$. Then $v$ solves the equation

$$
\partial_{t} v=\Delta v-\frac{2}{r} \partial_{r} v-(b \cdot \nabla) v-(\nabla v)^{2} .
$$

We will show that solutions to this equation satisfy one of the fundamental inequalities in the work of Nash.

First we define $\eta(x)$ to be smooth and radial with $\eta=1$ on $B_{1 / 2}$ and support in $B_{1}$. We rescale

$$
\eta_{R}(x)=\eta(x / R) R^{-3 / 2} .
$$

Further suppose that $\int_{\mathbb{R}^{3}} \eta^{2} d x=1$. Now we may define the weighted spaces

$$
\|f\|_{L^{p}\left(\eta_{R}\right)}^{p}=\int_{\mathbb{R}^{3}}|f|^{p} \eta_{R}^{2} d x .
$$

Now we may state the following lemma.

Lemma 5.2 Suppose that for some $0 \leq q<1$ we have

$$
\|b(s)\|_{L^{2}\left(\eta_{R}\right)} \leq C R^{-1+q}|s|^{-q / 2} .
$$

Then there is a $\delta>0$ such that

$$
-\int_{\mathbb{R}^{3}} \log u(x, t) \eta_{R}^{2} d x \leq C, \quad \text { for }-\delta R^{2} \leq t<0 .
$$

Notice that this implies the key step, equation (3.9) in [3], and thus proves the Hölder continuity. In fact, since (5.9) holds for every time, it is stronger than (3.9) which involves time integration. Further we remark that (4.2) is enough to ensure (5.8) with $q=2 \epsilon$ whenever $\epsilon>0$.

Proof. We first rescale by a factor $R$ for $x$ and $R^{2}$ for $t$. We define $v_{R}(x, t)=v\left(R x, R^{2} t\right)$, which satisfies

$$
\partial_{s} v_{R}=\Delta v_{R}-\frac{2}{r} \frac{\partial v_{R}}{\partial r}-\left(R b_{R} \cdot \nabla\right) v_{R}-\left(\nabla v_{R}\right)^{2}
$$

Above $b_{R}(x, t)=b\left(R x, R^{2} t\right)$. Our goal is now to prove that

$$
-\int_{\mathbb{R}^{3}} v_{R} \eta^{2} d x \leq C, \quad \text { for }-\delta \leq t<0 .
$$

The rescaled version of the assumption on $b$ becomes

$$
\left\|R b_{R}(\cdot, s)\right\|_{L^{2}(\eta)} \leq C|s|^{-q / 2} .
$$

Since we will only use (5.11), we shall drop all $R$ in the subscript from now on and set $R=1$. We have

$$
\int_{\mathbb{R}^{3}} \partial_{s} v \eta^{2} d x=\int_{\mathbb{R}^{3}}\left\{\Delta v-\frac{2}{r} \partial_{r} v-(b \cdot \nabla) v\right\} \eta^{2} d x-\int_{\mathbb{R}^{3}}(\nabla v)^{2} \eta^{2} d x .
$$

We will estimate the terms in parenthesis. 
For the first term we use the Cauchy-Schwartz inequality

$$
\int_{\mathbb{R}^{3}} \Delta v \eta^{2} d x=-2 \int_{\mathbb{R}^{3}} \nabla v \cdot \nabla \eta \eta d x \leq \frac{1}{8} \int_{\mathbb{R}^{3}}|\nabla v|^{2} \eta^{2} d x+8 \int_{\mathbb{R}^{3}}|\nabla \eta|^{2} d x .
$$

Next let

$$
\bar{v}:=v(s, x)-\langle v\rangle(s), \quad\langle v\rangle(s):=\int_{\mathbb{R}^{3}} v(s, x) \eta^{2} d x .
$$

We now consider the middle term inside the parenthesis. Integrating by parts, we have

$$
\begin{aligned}
-\int_{\mathbb{R}^{3}} \frac{2}{r} \partial_{r} v \eta^{2} d x=-\int_{\mathbb{R}^{3}} \frac{2}{r} \partial_{r} \bar{v} \eta^{2} d x & =-\left.\int_{-\infty}^{\infty} 2 \bar{v} \eta^{2} d z\right|_{r=0} ^{\infty}+\int_{\mathbb{R}^{3}} \frac{2}{r} \bar{v} \partial_{r} \eta^{2} d x \\
& \leq C-C\langle v\rangle(s)+4 \int_{\mathbb{R}^{3}} \bar{v} \frac{\partial_{r} \eta}{r} \eta d x .
\end{aligned}
$$

We have just used

$$
-\left.\int_{-\infty}^{\infty} 2 \bar{v} \eta^{2} d z\right|_{r=0} ^{\infty}=\left.\int_{-\infty}^{\infty} 2 \bar{v} \eta^{2} d z\right|_{r=0} \leq \int_{-\infty}^{\infty} 2 v(t, z, r=0) \eta^{2}(z, r=0) d z \leq C-C\langle v\rangle(s) .
$$

We remark that the constant is $u(t, z, r=0)=a \geq 1$. Furthermore,

$$
4 \int_{\mathbb{R}^{3}} \bar{v} \frac{\partial_{r} \eta}{r} \eta d x \leq 4\|\bar{v}\|_{L^{2}(\eta)}\left\|\partial_{r} \eta / r\right\|_{L^{2}\left(B_{1}\right)} \leq C+\frac{1}{8}\|\nabla v\|_{L^{2}(\eta)}^{2}
$$

Here we used the spectral gap estimate

$$
\int_{\mathbb{R}^{3}}|\nabla v|^{2} \eta^{2} d x \geq c \int_{\mathbb{R}^{3}} \bar{v}^{2} \eta^{2} d x .
$$

Finally we consider the last term in parenthesis. We use the Cauchy-Schwartz inequality together with (5.11) to obtain

$$
\int_{\mathbb{R}^{3}}(b \cdot \nabla) v \eta^{2} d x \leq\|b\|_{L^{2}(\eta)}\|\nabla v\|_{L^{2}(\eta)} \leq 4\|b\|_{L^{2}(\eta)}^{2}+\frac{1}{4}\|\nabla v\|_{L^{2}(\eta)}^{2} \leq C|s|^{-q}+\frac{1}{4}\|\nabla v\|_{L^{2}(\eta)}^{2} .
$$

Combining the inequalities in this paragraph we have

$$
\int_{\mathbb{R}^{3}}\left\{\Delta v-\frac{2}{r} \partial_{r} v-(b \cdot \nabla) v\right\} \eta^{2} d x-\frac{1}{2} \int_{\mathbb{R}^{3}}(\nabla v)^{2} \eta^{2} d x \leq C\left(1+|s|^{-q}-\langle v\rangle(s)\right) .
$$

Thus there is a constant $C$ such that

$$
\int_{\mathbb{R}^{3}} \partial_{s} v(s) \eta^{2} d x \leq C\left(1+|s|^{-q}-\langle v\rangle(s)\right)-\frac{1}{2} \int_{\mathbb{R}^{3}}|\nabla v|^{2} \eta^{2} d x .
$$

We will use this inequality to prove the lemma.

We plug the Nash inequality (5.7) with $M=2$ into the inequality above (also using the spectral gap estimate) to obtain

$$
\partial_{s}\langle v\rangle(s) \leq C\left(1+|s|^{-q}-\langle v\rangle(s)\right)-\frac{C\|u(s)\|_{L^{1}(\eta)}^{2}}{M^{2}}\left|\langle v\rangle(s)+\log \|u(s)\|_{L^{1}(\eta)}\right|^{2} .
$$


This differential inequality will now be manipulated into a form which we find useful. For some $\kappa>0,(5.6)$ lets us conclude

$$
\|u\|_{L^{1}\left(Q_{1 / 2}\right)} \geq \kappa
$$

Let $\chi$ be the characteristic function of the non-empty set

$$
W:=\left\{s:\|u(s)\|_{L^{1}\left(B_{1 / 2}\right)} \geq \kappa / 10\right\} .
$$

Since $u$ is bounded above by a constant $M$, it follows from (5.13) that

$$
|W| \geq \frac{\kappa}{10 M}
$$

Hence for some $O(1)$ constants $C \geq 1$ and $\gamma>0$ we have

$$
\begin{aligned}
\partial_{s}\langle v\rangle(s) \leq & C\left(1+|s|^{-q}-\langle v\rangle(s)\right)-C \chi(s)\|u(s)\|_{L^{1}(\eta)}^{2}\left|\langle v\rangle(s)+\log \|u(s)\|_{L^{1}(\eta)}\right|^{2} \\
& \leq C\left(1+|s|^{-q}-\langle v\rangle(s)\right)-\gamma \chi(s)\left|\langle v\rangle(s)+\log \|u(s)\|_{L^{1}(\eta)}\right|^{2} .
\end{aligned}
$$

Notice that, since $q<1$, this inequality implies for $s_{2} \geq s_{1}$ that

$$
\langle v\rangle\left(s_{2}\right) \leq e^{C\left|s_{1}-s_{2}\right|}\langle v\rangle\left(s_{1}\right)+C e^{C\left|s_{2}\right|} .
$$

Therefore we may assume that

$$
\langle v\rangle(s) \geq 4|\log (10 / \kappa)|+4 C\left(1+|s|^{-q}\right), \quad \text { for all }-1 \leq s \leq-\kappa /(20 M) .
$$

Since, otherwise, we would have $\langle v\rangle(s) \leq C_{1}$ for some $s_{0}$ in that range and then for all times later on. This would prove the Lemma.

Under assumption (5.16), we have for $-1 \leq s \leq-\kappa /(20 M)$ and some positive constant $C_{1}$ that

$$
\partial_{s}\langle v\rangle(s) \leq-C_{1} \chi(s)\langle v\rangle(s)^{2} .
$$

Divide both sides by $\langle v\rangle(s)^{2}$ and integrate the inequality from -1 to $t$. We have for $t=-\frac{\kappa}{20 M}$ the following

$$
\langle v\rangle(-1)^{-1}-\langle v\rangle(t)^{-1} \leq-\int_{-1}^{t} C_{1} \chi(s) d s \leq-C_{2} .
$$

Notice that the range of $t$ and (5.14) guarantee that $C_{2}>0$. Since by assumption (5.16), $\langle v\rangle(-1) \geq 0$, this proves (5.9) at time $t=-\frac{\kappa}{20 M}$ and hence all the time later on.

\subsection{Proof of Hölder continuity}

From Lemma 5.2, there is a $0<\tau<1$ such that for any $\varepsilon>0$ there is an $\delta$ so that

$$
|\{X \in Q(R, \tau): u(X) \leq \delta\}| \leq \epsilon|Q(R, \tau)|,
$$

Let $U=\delta-u$, where $\delta$ is the constant from (5.19). $U$ is clearly a solution of (5.1) and $\left.U\right|_{r=0}=\delta-a<0$. So we can apply Lemma 4.1 to conclude

$$
\sup _{Q(d / 2)}(\delta-u) \leq\left(\frac{C}{|Q(d)|} \int_{Q(d)}\left|(\delta-u)^{+}\right|^{2}\right)^{1 / 2} .
$$


Let $d=\sqrt{\tau} R$ so that $Q(d) \subset Q(R, \tau)$. By (5.20) and (5.19),

$$
\begin{aligned}
\delta-\inf _{Q(d / 2)} u & \leq\left(\frac{C}{|Q(d)|} \int_{Q(d)}\left|(\delta-u)^{+}\right|^{2}\right)^{1 / 2} \\
& \leq\left(\frac{C \delta^{2} \epsilon|Q(R, \tau)|}{|Q(d)|}\right)^{1 / 2}=C \delta \epsilon^{1 / 2}(\tau)^{-3 / 4},
\end{aligned}
$$

which is less than $\frac{\delta}{2}$ if $\epsilon$ is chosen sufficiently small. We conclude

$$
\inf _{Q(d / 2)} u \geq \frac{\delta}{2}
$$

This is the lower bound we seek.

We define

$$
m_{d} \equiv \inf _{Q(d / 2)} \Gamma, \quad M_{d} \equiv \sup _{Q(d / 2)} \Gamma
$$

Then from (5.2) we have

$$
\inf _{Q(d / 2)} u= \begin{cases}2\left(m_{d}-m_{2}\right) / M & \text { if }-m_{2}>M_{2}, \\ 2\left(M_{2}-M_{d}\right) / M & \text { else, }\end{cases}
$$

Notice that both expressions above are non-negative in any case; thus we can add them together to observe that

$$
\frac{\delta}{2} \leq \frac{2}{M}\{M-\operatorname{osc}(\Gamma, d / 2)\} .
$$

Here $\operatorname{osc}(\Gamma, d / 2)=M_{d}-m_{d}$ and $\operatorname{osc}(\Gamma, 2 R)=M_{2}-m_{2}=M$. We rearrange the above

$$
\operatorname{osc}(\Gamma, d / 2) \leq\left(1-\frac{\delta}{4}\right) \operatorname{osc}(\Gamma, 2 R)
$$

This is enough to produce the desired Hölder continuity via the standard argument.

\section{Proof of main theorem}

In this section we prove Theorem 1.1 under the assumption (1.5). It is similar to [3] section 2, which assumes a stronger assumption $|v| \leq C_{*}\left(r^{2}-t\right)^{-1 / 2}$. First we show that our solutions, which satisfy (1.5), are in fact suitable weak solutions. Recall that a pair of suitable weak solution $(v, p)$ satisfy

$$
v \in L_{t}^{\infty} L_{x}^{2}(Q), \quad \nabla v \in L^{2}(Q), \quad p \in L^{3 / 2}(Q) .
$$

and the local energy inequality.

Fix $\beta \in(1,5 / 3)$. For $t \in\left(-T_{0}, 0\right)$, we have by $(1.5)$

$$
\begin{aligned}
& \int_{\mathbb{R}^{3}} \frac{|v(x, t)|^{4}}{|x|^{\beta}} d x \leq \int_{\mathbb{R}^{3}} \frac{1}{|x|^{\beta}} \frac{C_{*} r d r d z}{\left(r^{2}-t\right)^{2-8 \varepsilon} r^{8 \varepsilon}|t|^{4 \varepsilon}}, \\
= & C \int_{\mathbb{R}^{2}} \frac{C_{*} r d r}{\left(r^{2}-t\right)^{2-8 \varepsilon} r^{\beta-1+8 \varepsilon}|t|^{4 \varepsilon}}=C C_{*}|t|^{-(1+\beta) / 2},
\end{aligned}
$$


where we have used the scaling and $\beta-1+8 \varepsilon<2$ so that it is integrable. Define $R_{i}$ 's to be the Riesz transforms: $R_{i}=\frac{\partial_{i}}{\sqrt{-\Delta}}$. We consider the singular integral

$$
\tilde{p}(x, t)=\int \sum_{i, j} \partial_{i} \partial_{j}\left(v_{i} v_{j}\right)(y) \frac{1}{4 \pi|x-y|} d y=\sum_{i, j} R_{i} R_{j}\left(v_{i} v_{j}\right) .
$$

Since $|x|^{-\beta}$ is a $A_{2}$ weight function, we have

$$
\int \frac{1}{|x|^{\beta}}|\tilde{p}(x, t)|^{2} d x \leq c \int \frac{1}{|x|^{\beta}}|v(x, t)|^{4} d x \leq c+c|t|^{-(1+\beta) / 2} .
$$

With this estimate, the same argument as in [3] proves that $p(x, t)=\tilde{p}(x, t)$ for all $x$ and for almost every $t$. Moreover, from (6.2) and $\beta<5 / 3$ we conclude that

$$
\int_{Q_{1}}|p(x, t)|^{3 / 2} d x d t \leq c \int_{-1}^{0}\left(\int_{B_{1}} \frac{1}{|x|^{\beta}}|p(x, t)|^{2} d x\right)^{3 / 4} d t \leq C .
$$

Since $\varepsilon$ is arbitrarily small, the pointwise estimate (1.5) on $v$ implies

$$
v \in L_{t}^{s} L_{x}^{q}\left(Q_{1}\right), \quad \frac{1}{q}+\frac{1}{s}>\frac{1}{2}, \quad q<\frac{1}{\varepsilon} .
$$

We will use $(s, q)=(3,3)$. Thus the vector product of (N-S) with $u \varphi$ for any $\varphi \in C_{c}^{\infty}\left(Q_{1}\right)$ is integrable in $Q_{1}$ and we can integrate by parts to get

$$
2 \int_{Q}|\nabla v|^{2} \varphi=\int_{Q}\left\{|v|^{2}\left(\partial_{t} \varphi+\Delta \varphi\right)+\left(|v|^{2}+2 p\right) v \cdot \nabla \varphi\right\}, \quad \forall \varphi \in C_{c}^{\infty}(Q), \varphi \geq 0 .
$$

For any $R \in(0,1)$ and $t_{0} \in\left(-R^{2}, 0\right)$, we can further choose a sequence of $\varphi$ which converges a.e. in $Q_{R}$ to the Heviside function $H\left(t_{0}-t\right)$. Since the limit of $\partial_{t} \varphi$ is the negative delta function in $t$, this gives us the estimate

$$
\underset{-R^{2}<t<0}{\operatorname{ess} \sup _{B_{R}}} \int_{B}|v(x, t)|^{2} d x+\int_{Q_{R}}|\nabla v|^{2} \leq C_{R} \int_{Q_{1}}\left(|v|^{3}+|p|^{3 / 2}\right) .
$$

These estimates show that $(v, p)$ is a suitable weak solution of (N-S) in $Q_{R}$. Note that these bounds depend on $C_{*}$ of $(1.5)$ only, not on $\|p\|_{L^{5 / 3}\left(\mathbb{R}^{3} \times\left(-T_{0}, 0\right)\right)}$.

To prove Theorem 1.1, it suffices to show that every point on the $z$-axis is regular. Suppose now a point $x_{*}=\left(0,0, x_{3}\right)$ on the $z$-axis is a singular point of $v$. Without loss of generality, we assume that $x_{3}=0$. We will use the following regularity criterion, a variant of the criterion in [1] and proven in [13], to obtain a contradiction.

Lemma 6.1 Suppose that $(v, p)$ is a suitable weak solution of $(\mathrm{N}-\mathrm{S})$ in $Q\left(X_{0}, 1\right)$. Then there exists an $\varepsilon_{1}>0$ so that $X_{0}$ is a regular point if

$$
\limsup _{R \downarrow 0} \frac{1}{R^{2}} \int_{Q\left(X_{0}, R\right)}|v|^{3} \leq \varepsilon_{1} .
$$


Let $\left(v^{\lambda}, p^{\lambda}\right)$ be the rescaled solutions of (N-S) defined by

$$
v^{\lambda}(x, t)=\lambda v\left(\lambda x, \lambda^{2} t\right), \quad p^{\lambda}(x, t)=\lambda^{2} p\left(\lambda x, \lambda^{2} t\right) .
$$

For $\left(v^{\lambda}, p^{\lambda}\right)$ with $0<\lambda<1$, the pointwise estimate (1.5) is preserved:

$$
\left|v^{\lambda}(x, t)\right| \leq C_{*}\left(r^{2}-t\right)^{-1 / 2+2 \varepsilon}|t|^{-\varepsilon} r^{-2 \varepsilon}, \quad(x, t) \in \mathbb{R}^{3} \times\left(-T_{0}, 0\right) .
$$

Fix $R_{*}>0$. Since we assume $x_{*}$ is a singular point, by Lemma 6.1 there is a sequence $\lambda_{k}, k \in \mathbb{N}$, so that $\lambda_{k} \rightarrow 0$ as $k \rightarrow \infty$ and

$$
\frac{1}{R_{*}^{2}} \int_{Q_{R_{*}}}\left|v^{\lambda_{k}}\right|^{3}=\frac{1}{\left(R_{*} \lambda_{k}\right)^{2}} \int_{Q\left(x_{*}, R_{*} \lambda_{k}\right)}|v|^{3}>\varepsilon_{1} .
$$

We will derive a contradiction to this statement.

Since $\left(v^{\lambda}, p^{\lambda}\right)$ satisfies the pointwise estimate (1.5), we have $v^{\lambda} \in L^{q}\left(Q_{1}\right)$ for $q \in(1,4)$. Moreover, the same argument as above provides the uniform bounds for $R<1$ :

$$
\underset{-R^{2}<t<0}{\operatorname{essup} \sup _{B_{R}}}\left|v^{\lambda}(x, t)\right|^{2} d x+\int_{Q_{R}}\left|\nabla v^{\lambda}\right|^{2} \leq c \int_{Q_{1}}\left|v^{\lambda}\right|^{3}+\left|p^{\lambda}\right|^{3 / 2} \leq C .
$$

Following the same argument in section 2.3 and section 2.4 of [3], we conclude from these estimates that there is a subsequence of $\left(v^{\lambda_{k}}, p^{\lambda_{k}}\right)$, still denoted by $\left(v^{\lambda_{k}}, p^{\lambda_{k}}\right)$, weakly converges to some suitable weak solution $(\bar{v}, \bar{p})$ of the Navier-Stokes equations in $Q_{R}$ and

$$
v^{\lambda_{k}} \rightarrow \bar{v},
$$

strongly in $L^{q}\left(Q_{R}\right)$ for all $1 \leq q<4$ and $0<R<1$.

Now the Hölder continuity of $\Gamma=r v_{\theta}$ at $t=0$ proven in Section 5 implies

$$
\int_{Q_{R}}\left|v_{\theta}^{\lambda}\right| \leq C \lambda^{\alpha} \rightarrow 0 \quad \text { as } \quad \lambda \downarrow 0
$$

for some $\alpha>0$. Thus the limit $\bar{v}$ has no-swirl, $\bar{v}_{\theta}=0$.

Let $\bar{\omega}=\nabla \times \bar{v}$ be the vorticity of $\bar{v}$ and $\bar{\omega}_{\theta}=\partial_{z} \bar{v}_{r}-\partial_{r} \bar{v}_{z}$ be the $\theta$ component of $\bar{\omega}$. The function $\Omega=\bar{\omega}_{\theta} / r$ solves

$$
\left(\partial_{t}+\bar{b} \cdot \nabla-\Delta-\frac{2}{r} \partial_{r}\right) \Omega=0,
$$

where $\bar{v}_{\theta}=0$ is used and $\bar{b}=\bar{v}_{r} e_{r}+\bar{v}_{z} e_{z}=\bar{v}$.

Since $\bar{v}$ is the limit of $v^{\lambda_{k}}$, it satisfies (1.5) and also satisfies (6.4). Following the argument of section 2.4 in [3], we conclude from (6.4) and the estimates for the Stokes system that

$$
\|\nabla \bar{v}\|_{L_{t}^{5 / 4} L_{x}^{5 / 2}\left(Q_{5 / 8}\right)} \leq C\|\bar{v}\|_{L_{t}^{5 / 2} L_{x}^{5}\left(Q_{3 / 4}\right)}^{2}+C\|\bar{v}\|_{L^{5 / 4}\left(Q_{3 / 4}\right)} \leq C .
$$

Hence $\Omega$ has the bound

$$
\|\Omega\|_{L^{20 / 19}\left(Q_{5 / 8}\right)} \leq\|\nabla \bar{v}\|_{L_{t}^{5 / 4} L_{x}^{5 / 2}\left(Q_{5 / 8}\right)}\|1 / r\|_{L_{t}^{\infty} L_{x}^{20 / 11}\left(Q_{5 / 8}\right)} \leq C .
$$

Since $\bar{b}$ satisfies (1.5), it also satisfies (4.2). We conclude from the local maximum estimate Lemma 4.1 and (6.12) that

$$
\Omega \in L^{\infty}\left(Q_{5 / 16}\right) .
$$


Furthermore we know that $\operatorname{curl} \bar{v}=\bar{\omega}_{\theta} e_{\theta} \in L^{\infty}\left(Q_{5 / 16}\right)$ from the above estimate on $\Omega$ since $\bar{v}_{\theta}=0$. Now we can apply the div-curl estimate

$$
\left\|\nabla^{k+1} v\right\|_{L^{q}\left(B_{R_{2}}\right)} \leq c\left\|\nabla^{k} \operatorname{div} v\right\|_{L^{q}\left(B_{R_{1}}\right)}+c\left\|\nabla^{k} \operatorname{curl} v\right\|_{L^{q}\left(B_{R_{1}}\right)}+c\|v\|_{L^{1}\left(B_{R_{1}}\right)},
$$

to obtain $L^{\infty}$ estimate for $\bar{v}$. Since $\operatorname{div} \bar{v}=0$ and $\bar{v} \in L_{t}^{\infty} L_{x}^{1}\left(Q_{5 / 16}\right)$ by (1.5), we thus conclude $\nabla \bar{v} \in$ $L_{t}^{\infty} L_{x}^{4}\left(Q_{1 / 4}\right)$ by taking $q=4$ and $k=0$ in the div-curl estimate. By the Sobolev embedding, we have $\bar{v} \in L^{\infty}\left(Q_{1 / 4}\right)$.

Now we can deduce regularity of the original solution from the regularity of the limit solution. We have shown that

$$
|\bar{v}(x, t)| \leq C_{*}^{\prime} \quad \text { in } \quad Q_{1 / 4} .
$$

Above $C_{*}^{\prime}$ depends upon $C_{*}$ but not on the subsequence $\lambda_{k}$. Since the constant can be tracked, we may initially choose $R_{*}$ sufficiently small to guarantee that

$$
\frac{1}{R_{*}^{2}} \int_{Q_{R_{*}}}|\bar{v}|^{3} \leq \varepsilon_{1} / 2
$$

where $\varepsilon_{1}$ is the small constant in Lemma 6.1. Since $v^{\lambda_{k}} \rightarrow \bar{v}$ strongly in $L^{3}$, for $k$ sufficiently large we have

$$
\frac{1}{R_{*}^{2}} \int_{Q_{R_{*}}}\left|v^{\lambda}\right|^{3} \leq \frac{1}{R_{*}^{2}} \int_{Q_{R_{*}}}|\bar{v}|^{3}+\frac{1}{R_{*}^{2}} \int_{Q_{R_{*}}}\left|v^{\lambda}-\bar{v}\right|^{3} \leq \varepsilon_{1}
$$

But this is a contradiction to (6.9). We have proved Theorem 1.1.

\section{Appendix: The case $\varepsilon=0$}

In this appendix we prove Theorem 1.1 under the assumption $|v(x, t)| \leq C_{*} r^{-1}$, the $\varepsilon=0$ case. The argument in this appendix was obtained after a preprint of [12] appeared. Note that this argument does not take scaling limits and all bounds are computable.

Let $M$ be the maximum of $|v|$ up to a fixed time $t_{1}$. We will derive an upper bound of $M$ in terms of $C_{*}$ and independent of $t_{1}$. We may assume $M>1$. Define

$$
v^{M}(X, T)=M^{-1} v\left(X / M, T / M^{2}\right), \quad X=\left(X_{1}, X_{2}, Z\right) .
$$

For $x=\left(x_{1}, x_{2}, z\right)$ and $X=\left(X_{1}, X_{2}, Z\right)$, let $r=\left(x_{1}^{2}+x_{2}^{2}\right)^{1 / 2}$ and $R=\left(X_{1}^{2}+X_{2}^{2}\right)^{1 / 2}$. We have the following estimates for all $r$ and $R$ for time $t \leq t_{1}$ and $T \leq M^{2} t_{1}$ :

$$
|v(x, t)| \leq C / r, \quad\left|v^{M}(X, T)\right| \leq C / R, \quad\left|\nabla^{k} v^{M}\right| \leq C_{k} .
$$

The last inequality follows from $\left\|v^{M}\right\|_{L^{\infty}} \leq 1$ for $t<t_{1}$ and the regularity theorem of Navier-Stokes equations. Its angular component (we omit the time dependence below) $v_{\theta}^{M}(R, Z)$ satisfies $v_{\theta}^{M}(0, Z)=0=\partial_{Z} v_{\theta}^{M}(0, Z)$ for all $Z$. By mean value theorem and (A.1), $\left|v_{\theta}^{M}(R, Z)\right| \leq C R$ and $\left|\partial_{Z} v_{\theta}^{M}(R, Z)\right| \leq C R$ for $R \leq 1$. Together with (A.1) for $R \geq 1$, we get

$$
\left|v_{\theta}^{M}\right| \leq C \min \left(R, R^{-1}\right), \quad\left|\partial_{Z} v_{\theta}^{M}\right| \leq C \min (R, 1), \quad \text { for } R>0 .
$$


By [3, Theorem 3.1], under the assumption $|v(x, t)| \leq C_{*} r^{-1}, \Gamma=r v_{\theta}$ satisfies $|\Gamma(r, z, t)| \leq C r^{\alpha}$ when $r$ is small, uniformly in $r$ and $t$, for some $C$ and small $\alpha>0$ depending on $C_{*}$. Thus, $\left|v_{\theta}^{M}(R, Z)\right| \leq C R^{-1+\alpha} M^{-\alpha}$ for $R>0$. From these estimates we have

$$
\frac{\left|\partial_{Z}\left(v_{\theta}^{M}\right)^{2}(R)\right|}{R^{2}} \leq \frac{C \min \left(R, R^{-1+\alpha} M^{-\alpha}\right) \cdot \min (R, 1)}{R^{2}} \leq \frac{C}{R^{3-\alpha} M^{\alpha}+1} .
$$

Consider now the angular component of the rescaled vorticity. Recall $\Omega=\omega_{\theta} / r$. Let

$$
f=\Omega^{M}(X, T)=\Omega\left(X / M, T / M^{2}\right) M^{-3}=\omega_{\theta}^{M}(X, T) / R .
$$

Since $\omega_{\theta}^{M}$ and $\nabla \omega_{\theta}^{M}$ are bounded by (A.1) and $\left.\omega_{\theta}^{M}\right|_{R=0}=0$, we have $|f| \leq C(1+R)^{-1}$. From the equation of $\omega_{\theta}$ (see (2.7)), we have

$$
\left(\partial_{T}-L\right) f=g, \quad L=\Delta+\frac{2}{R} \partial_{R}-b^{M} \cdot \nabla_{X},
$$

where $g=R^{-2} \partial_{Z}\left(v_{\theta}^{M}\right)^{2}$ and $b^{M}=v_{r}^{M} e_{R}+v_{z}^{M} e_{Z}$. Let $P(T, X ; S, Y)$ be the evolution kernel for $\partial_{T}-L$. By Duhamel's formula

$$
f(X, T)=\int P(T, X ; S, Y) f(Y, S) d Y+\int_{S}^{T} \int P(T, X ; \tau, Y) g(Y, \tau) d Y d \tau=: I+I I .
$$

By Carlen and Loss [4], in particular its equation (2.5), the kernel $P$ satisfies $P \geq 0, \int P(T, X ; S, Y) d Y=1$ and, using $\left\|b^{M}\right\|_{\infty}<1$,

$$
P(T, X ; S, Y) \leq C(T-S)^{-3 / 2} e^{-h(|X-Y|, T-S)}, \quad h(a, T)=C \frac{a^{2}}{T}\left(1-\frac{T}{a}\right)_{+}^{2} .
$$

Using $e^{-h(a, T)} \leq C e^{-C a / T}$ we get for $X=\left(X_{1}, X_{2}, X_{3}\right)$ and $Y=\left(Y_{1}, Y_{2}, Y_{3}\right)$

$$
P(T, X ; S, Y) \leq C(T-S)^{-3 / 2} e^{-C\left|X_{3}-Y_{3}\right| /(T-S)} .
$$

Here we only assert the spatial decay in the $X_{3}$ direction so that the proof of [4], where the term $R^{-1} \partial_{R}$ in $L$ is not present, needs no revision. With these bounds and Hölder inequality we get

$$
|I| \leq\left[\int P(T, X ; S, Y)|f(Y, S)|^{3} d Y\right]^{\frac{1}{3}} \leq\left[C(T-S)^{-\frac{3}{2}} \int e^{-C \frac{\left|X_{3}-Y_{3}\right|}{T-S}} \frac{R d R}{(1+R)^{3}} d Z\right]^{\frac{1}{3}} \leq C(T-S)^{-1 / 6},
$$

and

$$
|I I| \leq \int_{S}^{T} \int(T-\tau)^{-3 / 2} e^{-C \frac{\left|X_{3}-Y_{3}\right|}{T-\tau}} \frac{R d R}{R^{3-\alpha} M^{\alpha}+1} d Z d \tau \leq C(T-S)^{1 / 2} M^{-2 \alpha / 3} .
$$

Combining these two estimates and choosing $S=T-M^{\alpha}>-T_{0} M^{2}$ (hence $f$ is defined), we have $|f(X, T)| \leq$ $C M^{-\alpha / 6}$. Thus

$$
\left|\omega_{\theta}(x, t)\right| \leq\left|\omega_{\theta}^{M}\left(r M, z M, t M^{2}\right)\right| M^{2} \leq\left|\Omega^{M}\left(r M, z M, t M^{2}\right)\right| M^{2} r M \leq C M^{3-\alpha / 6} r .
$$

Therefore, we have

$$
\left|\omega_{\theta}(x, t)\right| \leq C M^{2-\alpha / 12}, \quad \text { for } r \leq M^{-1+\alpha / 12} .
$$


Let $b=v_{r} e_{r}+v_{z} e_{z}$ and $B_{\rho}\left(x_{0}\right)=\left\{x:\left|x-x_{0}\right|<\rho\right\}$. By (2.20) and (2.9), $b$ satisfies $-\Delta b=\operatorname{curl}\left(\omega_{\theta} e_{\theta}\right)$, and hence the following estimate with $p>1$ (see e.g., [7], Theorem 8.17)

$$
\sup _{B_{\rho}\left(x_{0}\right)}|b| \leq C\left(\rho^{-\frac{3}{p}}|| b \|_{L^{p}\left(B_{2 \rho}\left(x_{0}\right)\right)}+\rho \sup _{B_{2 \rho}\left(x_{0}\right)}\left|\omega_{\theta}\right|\right) .
$$

Let $\rho=M^{-1+\alpha / 24}, x_{0} \in\{(r, \theta, z): r<\rho\}$ and $1<p<2$. By the assumption $|v| \leq C / r$,

$$
\rho^{-\frac{3}{p}}\|b\|_{L^{p}\left(B_{2 \rho}\left(x_{0}\right)\right)} \leq C \rho^{-\frac{3}{p}}\|1 / r\|_{L^{p}\left(B_{2 \rho}\left(x_{0}\right)\right)} \leq \frac{C}{2-p} \rho^{-1} \leq C(p) M^{1-\alpha / 24} .
$$

This together with (A.6), (A.7) and the fact $\left|v_{\theta}\right|=M\left|v_{\theta}^{M}\right| \leq M C \min \left(R, R^{-1+\alpha} M^{-\alpha}\right)$ imply

$$
|v(x, t)| \leq C M^{1-\alpha / 24}, \quad \text { for } r \leq M^{-1+\alpha / 24} .
$$

On the other hand, the assumption $|v| \leq C / r$ implies $|v| \leq C M^{1-\alpha / 24}$ for $r \geq M^{-1+\alpha / 24}$. Since $M$ is the maximum of $v$, this gives an upper bound for $M$.

\section{Acknowledgments}

The authors would like to thank the National Center for Theoretical Sciences at Taipei and National Taiwan University for hosting part of our collaboration. The research of Chen is partly supported by the NSC grant 95-2115-M-002-008 (Taiwan). The research of Strain is partly supported by the NSF fellowship DMS0602513 (USA). The research of Tsai is partly supported by an NSERC grant (Canada). The research of Yau is partly supported by the NSF grants DMS-0602038 and DMS-0804279 (USA).

\section{References}

[1] L. Caffarelli, R. Kohn, and L. Nirenberg, Partial regularity of suitable weak solutions of the Navier-Stokes equations, Comm. Pure Appl. Math. 35 (1982), no. 6, 771-831. MR 673830 (84m:35097)

[2] Dongho Chae and Jihoon Lee, On the regularity of the axisymmetric solutions of the Navier-Stokes equations, Math. Z. 239 (2002), no. 4, 645-671. MR 1902055 (2003d:35205)

[3] Chiun-Chuan Chen, Robert M. Strain, Tai-Peng Tsai, and Horng-Tzer Yau, Lower bound on the blow-up rate of the axisymmetric Navier-Stokes equations, Int. Math. Res. Not. 2008 (2008), article ID: rnn016, 31 pages.

[4] Eric A. Carlen and Michael Loss, Optimal smoothing and decay estimates for viscously damped conservation laws, with applications to the 2-D Navier-Stokes equation. A celebration of John F. Nash, Jr., Duke Math. J. 81 (1995), no. 1, 135-157. MR 1381974 (96m:35199)

[5] Ennio De Giorgi, Sulla differenziabilità e l'analiticità delle estremali degli integrali multipli regolari, Mem. Accad. Sci. Torino. Cl. Sci. Fis. Mat. Nat. (3) 3 (1957), 25-43 (Italian). MR 0093649 (20 \#172)

[6] E. B. Fabes and D. W. Stroock, A new proof of Moser's parabolic Harnack inequality using the old ideas of Nash, Arch. Rational Mech. Anal. 96 (1986), no. 4, 327-338. MR 855753 (88b:35037)

[7] David Gilbarg and Neil S. Trudinger, Elliptic partial differential equations of second order, Springer-Verlag, Berlin, 1983. MR 0737190 (86c:35035)

[8] Jean Leray, Sur le mouvement d'un liquide visqueux emplissant l'espace., Acta Math. 63 (1934), 193-248 (French).

[9] Gary M. Lieberman, Second order parabolic differential equations, World Scientific Publishing Co. Inc., River Edge, NJ, 1996. MR 1465184 (98k:35003) 
[10] Jürgen Moser, A new proof of De Giorgi's theorem concerning the regularity problem for elliptic differential equations, Comm. Pure Appl. Math. 13 (1960), 457-468. MR 0170091 (30 \#332)

[11] J. Nash, Continuity of solutions of parabolic and elliptic equations, Amer. J. Math. 80 (1958), 931-954. MR 0100158 (20 \#6592)

[12] G. Koch, N. Nadirashvili, G. Seregin, and V. Sverak, Liouville theorems for the Navier-Stokes equations and applications, preprint: http://arxiv.org/abs/0709.3599.

[13] Gang Tian and Zhouping Xin, Gradient estimation on Navier-Stokes equations, Comm. Anal. Geom. 7 (1999), no. 2, 221-257. MR 1685610 (2000i:35166) 